\title{
Metabolic interaction between cancer cells and stromal cells according to breast cancer molecular subtype
}

\author{
Junjeong Choi ${ }^{1}$, Do Hee Kim², Woo Hee Jung ${ }^{2}$ and Ja Seung Koo ${ }^{2^{*}}$
}

\begin{abstract}
Introduction: The aim of this study was to investigate the differential expression of markers related to metabolic, mitochondrial and autophagy status in different molecular subtypes of breast cancer.

Methods: Using tissue microarray sections generated from 740 cases of breast cancer, we performed immunohistochemical staining for Glut-1, CAIX, MCT4, ATP synthase, glutaminase, BNIP3, Beclin-1, LC3A, LC3B and p62. Based on the immunohistochemical expression of estrogen receptor (ER), progesterone (PR), HER2, and Ki-67 labeling index, the cases were classified into luminal A, luminal B, HER2 and triple-negative breast cancer (TNBC). We further classified metabolic phenotypes of tumors according to glycolytic status by assessing Glut-1 and CAIX expression as follows: Warburg type: tumor (glycolysis type), stroma (nonglycolysis type); reverse Warburg type: tumor (nonglycolysis type), stroma (glycolysis type); mixed type: tumor (glycolysis type), stroma (glycolysis type); and null type: tumor (nonglycolysis type), stroma (nonglycolysis type).

Results: Expression of Glut-1, MCT4 and LC3A was highest in TNBC and lowest in luminal A type $(P<0.001)$. Tumors were classified into 298 Warburg type (40.3\%), 54 reverse Warburg type (7.3\%), 62 mixed type (8.4\%) and 326 null type (44.0\%). The mixed type had a higher histologic grade, ER negativity, PR negativity and Ki-67 index, whereas the null type showed lower histologic grade, ER positivity, PR positivity and Ki-67 index $(P<0.001)$. TNBC constituted the major portion of Warburg and mixed types, and luminal A consisted mainly of reverse Warburg and null types $(P<0.001)$.

Conclusion: Breast cancer is heterogeneous in its metabolic status, and therefore it can be classified into various metabolic phenotypes. Specifically, the Warburg and mixed types had strong associations with TNBC, whereas reverse the Warburg and null types had associations with the luminal type, suggesting a correlation between metabolic phenotype and the biology of breast cancer.
\end{abstract}

\section{Introduction}

The metabolism of malignant tumors is generally explained by the Warburg effect theory, which describes the metabolic shift from mitochondrial oxidative phosphorylation (OXPHOS) to glycolysis in tumors [1]. Breast cancer is known to be heterogeneous, and the interaction between tumor cells and adjacent stroma is expected to have significant roles in tumor growth and progression. This kind of complex interaction may also exist in the metabolic processes of the tumor. Previous studies suggest a unique metabolic interaction between tumor cells and the stroma of breast cancer, known as the reverse Warburg effect theory [2-5]. According to this theory, reactive oxygen species (ROS), such as nitric oxide $(\mathrm{NO})$, generated by tumor cells bring oxidative stress to the stromal cells, leading to mitochondrial dysfunction, autophagy (mitophagy) and increased aerobic glycolysis through hypoxia-inducible factor $1 \alpha$ (HIF-1 $\alpha)$ and nuclear factor $\kappa B(\mathrm{NF}-\kappa \mathrm{B})$. Lactate generated by stromal cell glycolysis enters tumor cells and promotes

\footnotetext{
* Correspondence: kjs1976@yuhs.ac

${ }^{2}$ Department of Pathology, Yonsei University College of Medicine, 50 Yonsei-ro,

Seodaemun-gu, Seoul 120-752, South Korea

Full list of author information is available at the end of the article
} 
tumor cell growth and survival through efficient generation of ATP by OXPHOS in the mitochondria. Cancerassociated fibroblasts (CAFs), stromal cells with loss of caveolin-1 expression, have been implicated in this interaction in breast cancer because loss of caveolin-1 results from increased proteolysis by autophagy [3,5-7]. In addition to the reverse Warburg effect theory, it has been reported that certain types of tumor cells generate ATP through glycolysis as well as OXPHOS, which suggests various features of tumor metabolism $[8,9]$. Proteins involved in metabolism, mitochondrial function and autophagy may be differentially expressed in both tumor and stromal cells according to the aforementioned theories. These differences are summarized in Table 1.

Because breast cancer is heterogeneous with respect to clinical, histopathological and molecular features, several subclassifications have been investigated to stratify tumors with similar characteristics. Gene expression profiles have enabled molecular classification of tumors into luminal $\mathrm{A}$, luminal B, HER2, normal breast-like and basal-like types [10-12]. Studies have also revealed differences in histological and clinical manifestation between different molecular subtypes, such as therapeutic response and prognosis. We hypothesized that the metabolic interaction between tumor cells and stroma may differ according to the molecular subtypes of breast cancer. Because there are limited studies regarding this question, the aim of our present study was designed to investigate the differential expression of markers for metabolic, mitochondrial and autophagy status in different molecular subtypes of breast cancer.

\section{Methods}

\section{Patient selection}

Patients diagnosed with invasive breast cancer treated by surgical resection during the period from January 2002 to December 2006 were included in this study. Patients who received preoperative neoadjuvant chemotherapy or hormonal treatment were excluded. This study was approved by the Institutional Review Board (IRB) of Yonsei University Severance Hospital. The IRB exempted the informed consent from patients. A breast pathologist (JSK) retrospectively reviewed the histology of all cases using hematoxylin and eosin (H\&E)-stained slides. The histological grade was assessed using the Nottingham grading system [13]. Clinicopathologic parameters evaluated in each case included patient age at initial diagnosis, lymph node metastasis, tumor recurrence, distant metastasis and patient survival.

\section{Tissue microarray}

On H\&E-stained slides of tumors, a representative area was selected and the corresponding spot was marked on the surface of the paraffin block. Using a biopsy needle, the selected area was punched out and a 3-mm tissue core was placed into a $6 \times 5$ recipient block. Tissue from the invasive tumor was then extracted. More than two tissue cores were extracted to minimize extraction bias. Each tissue core was assigned a unique tissue microarray (TMA) location number that was linked to a database containing other clinicopathologic data.

\section{Immunohistochemistry}

The antibodies used for immunohistochemistry (IHC) in this study are shown in Table 2. Formalin-fixed, paraffinembedded (FFPE) tissue sections from the TMA were prepared for IHC. Briefly, 5- $\mu$ m-thick sections were obtained using a microtome, transferred into adhesive slides and dried at $62^{\circ} \mathrm{C}$ for $30 \mathrm{~min}$. After incubation with primary antibodies, immunodetection was performed with biotinylated anti-mouse immunoglobulin, followed by peroxidase-labeled streptavidin using a labeled streptavidin biotin kit with 3,3'-diaminobenzidine chromogen as the substrate. The primary antibody incubation step was omitted in the negative control. A positive control was included for each experiment: glucose transporter 1 (Glut-1): esophageal carcinoma; carbonic anhydrase IX (CAIX): renal carcinoma; monocarboxylate transporter 4 (MCT4): BCL2/ adenovirus E1B 19-kDa interacting protein 3 (BNIP3), kidney tissue; Beclin-1: breast tissue; microtubule-associated protein 1 light chain $3 \alpha$ (LC3A): brain tissue; microtubuleassociated protein 1 light chain $3 \beta$ (LC3B): brain tissue, p62: spleen tissue; ATP synthase: heart tissue; and glutaminase: liver tissue. Slides were counterstained with Harris hematoxylin.

\section{Interpretation of immunohistochemical staining}

All immunohistochemical markers were assessed by light microscopy. Pathologic parameters such as ER, PR and

Table 1 Comparison of metabolism type, mitochondrial status and autophagy status between the Warburg effect theory and reverse Warburg effect theory ${ }^{a}$

\begin{tabular}{llll}
\hline & Warburg effect theory & \multicolumn{2}{c}{ Reverse Warburg effect theory } \\
\cline { 3 - 4 } & Cancer cell & Cancer cell & Stromal cell \\
\hline Metabolism & Glycolysis & OXPHOS & Glycolysis \\
Mitochondrial status & Dysfunctional & Functional & Dysfunctional \\
Autophagy status & Not included & Not activated & Activated \\
\hline
\end{tabular}

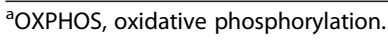


Table 2 Source, clone and dilution of antibodies used in this study ${ }^{a}$

\begin{tabular}{llll}
\hline Antibody & Clone & Dilution & Company \\
\hline Molecular subtype-related & SP1 & $1: 100$ & Thermo Scientific, Waltham, MA, USA \\
ER & PgR & $1: 50$ & Dako Denmark AS, Glostrup, Denmark \\
PR & Polyclonal & $1: 1,500$ & Dako Denmark AS, Glostrup, Denmark \\
HER2 & MIB-1 & $1: 150$ & Dako Denmark AS, Glostrup, Denmark \\
Ki-67 & & & \\
Glycolysis-related & SPM498 & $1: 200$ & Abcam, Cambridge, UK \\
Glut-1 & Polyclonal & $1: 100$ & Abcam, Cambridge, UK \\
CAIX & Polyclonal & $1: 100$ & Santa Cruz Biotechnology, Santa Cruz, CA, USA \\
MCT4 & & & \\
Mitochondrial status-related & Ana40 & $1: 100$ & Abcam, Cambridge, UK \\
BNIP3 & & & \\
Mitochondrial metabolism-related & 15H4C4 & $1: 100$ & Abcam, Cambridge, UK \\
ATP synthase & Polyclonal & $1: 100$ & Abcam, Cambridge, UK \\
Glutaminase & & & \\
Autophagy-related & Polyclonal & $1: 100$ & Abcam, Cambridge, UK \\
Beclin-1 & EP1528Y & $1: 100$ & Abcam, Cambridge, UK \\
LC3A & Polyclonal & $1: 100$ & Abcam, Cambridge, UK \\
LC3B & SQSTM1 & $1: 100$ & Abcam, Cambridge, UK \\
p62 & CAX, & &
\end{tabular}

${ }^{a}$ BNIP3, BCL2/adenovirus E1B 19-kDa interacting protein 3; CAIX, carbonic anhydrase IX; ER, estrogen receptor; Glut-1, glucose transporter 1; LC3A, microtubule-associated protein 1 light chain 3a; LC3B, microtubule-associated protein 1 light chain 3ß; MCT4, monocarboxylate transporter 4; PR, progesterone receptor.

HER2 expression were obtained from each patient's pathologic report. A cutoff value of $1 \%$ or more positively stained nuclei was used to define ER and PR positivity [14]. HER2 staining was analyzed according to the American Society of Clinical Oncology (ASCO)/College of American Pathologists (CAP) guidelines using the following categories: 0 = no immunostaining; $1+$ = weak, incomplete membranous staining, less than $10 \%$ of tumor cells; $2+=$ complete membranous staining, either uniform or weak in at least $10 \%$ of tumor cells; and $3+=$ uniform intense membranous staining in at least $30 \%$ of tumor cells [15]. HER2 immunostaining was considered positive when strong (3+) membranous staining was observed, whereas cases with 0 to $1+$ were regarded as negative. Cases showing 2+ HER2 expression were evaluated for HER2 amplification by fluorescence in situ hybridization (FISH).

Glut-1, CAIX, BNIP3, MCT4, Beclin-1, LC3A, LC3B and p62 immunohistochemical staining was evaluated on the basis of the proportion of stained cells and immunostaining intensity. The proportion of stained cells was graded 0 (negative), 1 (less than $30 \%$ positive) or 2 (more than $30 \%$ positive). Immunostaining intensity was graded as 0 (negative), 1 (weak), 2 (moderate) or 3 (strong). The scores for the proportion of stained cells and staining intensity were multiplied to provide a total score: negative ( 0 or 1 ) or positive (2 through 6 ). Ki-67 labeling indices (LIs) were scored by counting the number of positively stained nuclei and expressed as a percentage of total tumor cells.

\section{Fluorescence in situ hybridization analysis}

Before FISH analysis, invasive tumors were examined on H\&E-stained slides. FISH was subsequently performed on the confirmed tumor. FISH was performed using the PathVysion HER-2 DNA Probe Kit (Abbott Molecular, Abbott Park, IL, USA) according to the manufacturer's instructions. HER2 gene copy number on the slides was evaluated using an epifluorescence microscope (Olympus, Tokyo, Japan). At least 60 tumor cell nuclei in three separate regions were investigated for HER2 and chromosome 17 signals. HER2 gene amplification was determined according to the ASCO/CAP guidelines [15]. An absolute HER2 gene copy number lower than 4 or a HER2 gene/ chromosome 17 (chr17) copy number ratio (HER2/chr17 ratio) less than 1.8 was considered HER2-negative. An absolute HER2 copy number between 4 and 6 or a HER2/ chr17 ratio between 1.8 and 2.2 was considered HER2equivocal. An absolute HER2 copy number greater than 6 or a HER2/chr17 ratio higher than 2.2 was considered HER2-positive.

\section{Tumor phenotype classification}

In this study, we classified breast cancer phenotypes according to the IHC results for ER, PR, HER2 and Ki-67 
LI. FISH results for HER2 were as follows [16]: luminal A type: ER- and/or PR-positive, HER2-negative Ki-67 LI less than 14\%; luminal B type: (HER2-negative) ER- and/or PR-positive, HER2-negative and Ki-67 LI greater than or equal to $14 \%$ and (HER2-positive) ER- and/or PR-positive and HER2 overexpressed and/or amplified; HER2 type: ERand PR-negative and HER2 overexpressed and/or amplified; TNBC type: ER-, PR- and HER2-negative.

\section{Classification of tumor metabolic subtypes}

We also classified cases based on the results of immunohistochemical staining for metabolism-related proteins as follows: glycolysis type: Glut-1- and/or CAIX-positive; nonglycolysis type: Glut-1- and CAIX-negative; dysfunctional mitochondrial type: BNIP3-positive $[17,18]$; functional mitochondrial type: BNIP3-negative; activated autophagy type: positive for two or more markers from among Beclin-1, LC3A, LC3B and p62; and nonactivated autophagy type: positive for less than two markers from among Beclin-1, LC3A, LC3B and p62. We further classified the metabolic phenotypes of breast cancer as follows: Warburg type: tumor (glycolysis type), stroma (nonglycolysis type); reverse Warburg type: tumor (nonglycolysis type), stroma (glycolysis type); mixed type: tumor (glycolysis type), stroma (glycolysis); and null type: tumor (nonglycolysis type), stroma (non-glycolysis type).

\section{Laser microdissection and protein extraction from formalin-fixed, paraffin-embedded tissues}

To acquire tumors and tumor stroma, laser microdissection was performed with hematoxylin-stained, uncovered slides generated with FFPE blocks (LMD 6500; Leica, Wetzlar, Germany). Five cases per molecular subtype of breast cancer were microdissected. Protein extraction from microdissected FFPE tissues was performed using the Qproteome FFPE Tissue Kit (QIAGEN, Hilden, Germany). Briefly, microdissected FFPE tissues were deparaffinized in xylene and rehydrated in a graded series of alcohol. Afterward, the samples were mixed with FFPE extraction buffer EXB Plus $(100 \mu \mathrm{l}$ per sample; QIAGEN), incubated at $100^{\circ} \mathrm{C}$ for $20 \mathrm{~min}$, at $80^{\circ} \mathrm{C}$ for $2 \mathrm{~h}$ and then centrifuged for $15 \mathrm{~min}$ at $14,000 \times g$ at $4^{\circ} \mathrm{C}$. The protein concentrations in the supernatant were determined using the Bradford assay (Bio-Rad Laboratories, Hercules, CA, USA).

\section{Western blot analysis}

Total protein $(20 \mu \mathrm{g})$ from each sample was mixed with Laemmli sample buffer and heated at $100^{\circ} \mathrm{C}$ for $5 \mathrm{~min}$. It was then loaded into individual wells, resolved by 8\% SDS-PAGE and electroblotted onto nitrocellulose membranes (GE Healthcare Life Sciences, Pittsburgh, PA, USA). Membranes were blocked in 5\% nonfat dry milk in Tris-buffered saline with Tween 20 (TBS-T), then incubated with antibodies to Glut-1, CAIX, ATP synthase, glutaminase, MCT-4, LC3A and p62 overnight at $4^{\circ} \mathrm{C}$. The membranes were washed with TBS-T and then probed with peroxidase-conjugated goat anti-rabbit immunoglobulin G (1:2,000; Santa Cruz Biotechnology, Santa Cruz, CA, USA) for $1 \mathrm{~h}$ at room temperature. Washing was repeated and the membranes were developed with an enhanced chemiluminescence agent (Amersham/ GE Healthcare Life Sciences, Little Chalfont, UK). Band densities were measured using TINA image software (raytest, Straubenhardt, Germany).

\section{Statistical analyses}

Data were processed using SPSS for Windows version 12.0 software (SPSS Inc, Chicago, IL, USA). Student's $t$-test and Fisher's exact test were used to examine any differences in continuous and categorical variables, respectively. Significance was assumed when $P<0.05$. KaplanMeier survival curves and logrank statistics were employed to evaluate time to tumor recurrence and time to survival. Multivariate regression analysis was performed using the Cox proportional hazards model.

\section{Results}

Patients' characteristics according to tumor phenotype

The clinicopathologic characteristics of 740 patients, comprising 298 (40.3\%) cases of luminal A, 166 (22.4\%) cases of luminal B, 69 (9.3\%) cases of HER2 type and 207 (28\%) cases of TNBC type, are summarized in Table 3. TNBC type had the highest histologic grade, tumor stage and Ki-67 LI $(P<0.001, P=0.002$ and $P<0.001$, respectively). In addition, HER2 and TNBC types had higher incidences of tumor recurrence and patient death than other types $(P<0.001)$.

\section{Expression of metabolism-related proteins according to tumor phenotype}

The differential expression of metabolism-related proteins according to breast cancer phenotype is summarized in Table 4. Tumor expression of Glut-1, MCT4 and LC3A was highest in TNBC and lowest in the luminal A type $(P<0.001)$. Stromal expression of CAIX and MCT4 and tumor expression of cytoplasmic p62 was highest in HER2 type and lowest in luminal A type $(P=0.032$, $P<0.001$ and $P<0.001$, respectively). Tumor expression of CAIX and LC3B was highest in TNBC and lowest in luminal B type ( $P=0.008$ and $P=0.013$, respectively). HER2 type showed the highest tumor and stromal ATP synthase expression $(P=0.027$ and $P<0.001$, respectively) and stromal glutaminase expression $(P=0.001)$, whereas luminal A type showed the lowest expression of those markers. Expression of stromal LC3A and tumor expression of nuclear p62 were highest in luminal A and lowest in TNBC $(P<0.001)$. 
Table 3 Clinicopathologic characteristics of patients according to breast cancer phenotype ${ }^{a}$

\begin{tabular}{|c|c|c|c|c|c|c|}
\hline Parameters & $\begin{array}{c}\text { Total } \\
(N=740)(\%)\end{array}$ & $\begin{array}{c}\text { Luminal A } \\
(n=298)(\%)\end{array}$ & $\begin{array}{l}\text { Luminal B } \\
(n=166)(\%)\end{array}$ & $\begin{array}{c}\text { HER2 } \\
(n=69)(\%)\end{array}$ & $\begin{array}{c}\text { TNBC } \\
(n=207)(\%)\end{array}$ & $P$-value \\
\hline Age (years, mean \pm SD) & $49.7 \pm 11.0$ & $50.6 \pm 10.5$ & $48.5 \pm 10.1$ & $52.8 \pm 9.8$ & $48.4 \pm 12.4$ & 0.007 \\
\hline Histologic grade & & & & & & $<0.001$ \\
\hline । & $118(15.9)$ & $90(30.2)$ & $18(10.8)$ & $1(1.4)$ & $9(4.3)$ & \\
\hline$\|$ & $373(50.4)$ & $180(60.4)$ & $90(54.2)$ & $35(50.7)$ & $68(32.9)$ & \\
\hline III & $249(33.6)$ & $28(9.4)$ & $58(34.9)$ & $33(47.8)$ & $130(62.8)$ & \\
\hline Tumor stage & & & & & & 0.002 \\
\hline $\mathrm{T} 1$ & $358(48.4)$ & $166(55.7)$ & $86(51.8)$ & $31(44.9)$ & $75(36.2)$ & \\
\hline $\mathrm{T} 2$ & $367(49.6)$ & $125(41.9)$ & $78(47.0)$ & $37(53.6)$ & $127(61.4)$ & \\
\hline T3 & $15(2.0)$ & $7(2.3)$ & $2(1.2)$ & $1(1.4)$ & $5(2.4)$ & \\
\hline Nodal stage & & & & & & 0.041 \\
\hline NO & $436(58.9)$ & $168(56.4)$ & $91(54.8)$ & $42(60.9)$ & $135(65.2)$ & \\
\hline N1 & $200(27.0)$ & $90(30.2)$ & $43(25.9)$ & $13(18.8)$ & $54(26.1)$ & \\
\hline $\mathrm{N} 2$ & $66(8.9)$ & $27(9.1)$ & $17(18.5)$ & $10(14.5)$ & $12(5.8)$ & \\
\hline N3 & $38(5.1)$ & $13(4.4)$ & $15(9.0)$ & $4(5.8)$ & $6(2.9)$ & \\
\hline Estrogen receptor status & & & & & & $<0.001$ \\
\hline Negative & $286(38.6)$ & $5(1.7)$ & $5(3.0)$ & $69(100.0)$ & $207(100.0)$ & \\
\hline Positive & $454(61.4)$ & $293(98.3)$ & $161(97.0)$ & $0(0.0)$ & $0(0.0)$ & \\
\hline Progesterone receptor status & & & & & & $<0.001$ \\
\hline Negative & $371(50.1)$ & $50(16.8)$ & $46(27.7)$ & $69(100.0)$ & $207(100.0)$ & \\
\hline Positive & $369(49.9)$ & $248(83.2)$ & $120(72.3)$ & $0(0.0)$ & $0(0.0)$ & \\
\hline HER2 status & & & & & & $<0.001$ \\
\hline 0 & $290(39.2)$ & $108(36.2)$ & $23(13.9)$ & $0(0.0)$ & $159(76.8)$ & \\
\hline $1+$ & $186(25.1)$ & $118(39.6)$ & $33(20.0)$ & $0(0.0)$ & $35(16.9)$ & \\
\hline $2+$ & $142(19.2)$ & $72(24.2)$ & $41(24.7)$ & $16(23.2)$ & $13(6.3)$ & \\
\hline $3+$ & $122(16.5)$ & $0(0.0)$ & $69(41.6)$ & $53(76.8)$ & $0(0.0)$ & \\
\hline Ki-67 LI (\%, mean \pm SD) & $18.1 \pm 19.2$ & $4.7 \pm 3.7$ & $19.7 \pm 12.7$ & $19.5 \pm 12.5$ & $35.6 \pm 23.7$ & $<0.001$ \\
\hline Tumor recurrence & $69(9.3)$ & $15(5.0)$ & $12(7.2)$ & $11(15.9)$ & $31(15.0)$ & $<0.001$ \\
\hline Patient's death & $67(9.1)$ & $14(4.7)$ & $11(6.6)$ & $12(17.4)$ & $30(14.5)$ & $<0.001$ \\
\hline Duration of clinical follow-up (months, mean \pm SD) & $70.2 \pm 31.7$ & $72.7 \pm 30.0$ & $70.3 \pm 30.3$ & $67.1 \pm 35.8$ & $67.8 \pm 33.8$ & 0.291 \\
\hline
\end{tabular}

aLI, labeling index; TNBC triple-negative breast cancer.

\section{Correlation between metabolism-related proteins and clinicopathologic factors}

The correlation between expression of metabolism-related proteins and clinicopathologic parameters is summarized in Table 5. Tumor expression of Glut-1 was associated with higher histologic grade $(P<0.001)$, ER negativity $(P<0.001)$, higher T stage $(P<0.001)$ and higher Ki67 LI $(P<0.001)$, whereas CAIX was associated with higher Ki-67 LI $(P<0.001)$. Stromal ATP synthase expression was associated with HER 2 positivity $(P<0.001)$, and stromal glutaminase expression was associated with higher KI-67 LI $(P=0.021)$. Tumor expression of MCT4 was associated with higher histologic grade $(P<0.001)$, ER negativity $(P<0.001)$, PR negativity $(P<0.001)$, higher T stage $(P<0.001)$ and higher Ki-67 LI $(P<0.001)$.
Stromal expression of MCT4 was associated with higher histologic grade $(P<0.001)$, ER negativity $(P<0.001)$, PR negativity $(P<0.001)$, HER2 positivity $(P<0.001)$ and higher Ki-67 LI $(P<0.001)$. Tumor expression of LC3A was associated with higher histologic grade $(P<0.001)$, ER negativity $(P<0.001)$, PR negativity $(P<0.001)$, HER2 negativity $(P<0.001)$ and higher Ki-67 LI $(P<0.001)$. In contrast, stromal expression of LC3A was associated with ER positivity $(P<0.001)$, PR positivity $(P<0.001)$ and lower Ki-67 LI $(P=0.032)$. Tumor expression of cytoplasmic p62 was associated with HER2 positivity $(P<0.001)$, whereas nuclear p62 was associated with lower histologic grade $(P<0.001)$, ER positivity $(P<0.001)$, PR positivity $(P<0.001)$ and lower Ki-67 LI $(P<0.001)$. 
Table 4 Expression of metabolism-related proteins according to breast cancer phenotype ${ }^{a}$

\begin{tabular}{|c|c|c|c|c|c|c|}
\hline Parameters & $\begin{array}{l}\text { Total } \\
(N=740)(\%)\end{array}$ & $\begin{array}{l}\text { Luminal A } \\
(n=298)(\%)\end{array}$ & $\begin{array}{l}\text { Luminal B } \\
(n=166)(\%)\end{array}$ & $\begin{array}{l}\text { HER2 } \\
(n=69)(\%)\end{array}$ & $\begin{array}{l}\text { TNBC } \\
(n=207)(\%)\end{array}$ & $P$-value \\
\hline Glut-1 in tumor & & & & & & $<0.001$ \\
\hline Negative & $504(68.1)$ & $260(87.2)$ & $124(74.7)$ & $47(68.1)$ & $73(35.3)$ & \\
\hline Positive & $236(31.9)$ & $38(12.8)$ & $42(25.3)$ & $22(31.9)$ & $134(64.7)$ & \\
\hline Glut-1 in stroma & & & & & & 0.103 \\
\hline Negative & $724(97.8)$ & $296(99.3)$ & $162(97.6)$ & $66(95.7)$ & $200(96.6)$ & \\
\hline Positive & $16(2.2)$ & $2(0.7)$ & $4(2.4)$ & $3(4.3)$ & $7(3.4)$ & \\
\hline CAIX in tumor & & & & & & 0.008 \\
\hline Negative & $520(70.3)$ & $217(72.8)$ & $127(76.5)$ & $49(71.0)$ & $127(61.3)$ & \\
\hline Positive & $220(29.7)$ & $81(27.2)$ & $39(23.5)$ & $20(29.0)$ & $80(38.6)$ & \\
\hline CAIX in stroma & & & & & & 0.032 \\
\hline Negative & $627(84.7)$ & $264(88.6)$ & $137(82.5)$ & $52(75.4)$ & $174(84.1)$ & \\
\hline Positive & $113(15.3)$ & $34(11.4)$ & $29(17.5)$ & 17 (24.6) & $33(15.9)$ & \\
\hline ATP synthase in tumor & & & & & & 0.027 \\
\hline Negative & $30(4.1)$ & $20(6.7)$ & $4(2.4)$ & $1(1.4)$ & $5(2.4)$ & \\
\hline Positive & $710(95.9)$ & $278(93.3)$ & $162(97.6)$ & 68 (98.6) & $202(97.6)$ & \\
\hline ATP synthase in stroma & & & & & & $<0.001$ \\
\hline Negative & $570(77.0)$ & $256(85.9)$ & $112(67.5)$ & $38(55.1)$ & $164(79.2)$ & \\
\hline Positive & $170(23.0)$ & $42(14.1)$ & $54(32.5)$ & $31(44.9)$ & $43(20.8)$ & \\
\hline Glutaminase in tumor & & & & & & 0.164 \\
\hline Negative & $219(29.6)$ & $85(28.5)$ & $60(36.1)$ & $21(30.4)$ & $53(25.6)$ & \\
\hline Positive & $521(70.4)$ & $213(71.5)$ & $106(63.9)$ & $48(69.6)$ & $154(74.4)$ & \\
\hline Glutaminase in stroma & & & & & & 0.001 \\
\hline Negative & $495(66.9)$ & $223(74.8)$ & $105(63.3)$ & $39(56.5)$ & $128(61.8)$ & \\
\hline Positive & $245(33.1)$ & $75(25.2)$ & $61(36.7)$ & $30(43.5)$ & $79(38.2)$ & \\
\hline BNIP3 in tumor & & & & & & 0.262 \\
\hline Negative & $504(68.1)$ & $206(69.1)$ & $112(67.5)$ & $40(58.0)$ & $146(70.5)$ & \\
\hline Positive & $236(31.9)$ & $92(30.9)$ & $54(32.5)$ & $29(42.0)$ & $61(29.5)$ & \\
\hline BNIP3 in stroma & & & & & & 0.262 \\
\hline Negative & $700(94.6)$ & $281(94.3)$ & $159(95.8)$ & $62(89.9)$ & $198(95.7)$ & \\
\hline Positive & $40(5.4)$ & $17(5.7)$ & $7(4.2)$ & $7(10.1)$ & $9(4.3)$ & \\
\hline MCT4 in tumor & & & & & & $<0.001$ \\
\hline Negative & $540(73.0)$ & $253(84.9)$ & $118(71.1)$ & $49(71.0)$ & $120(58.0)$ & \\
\hline Positive & $200(27.0)$ & $45(15.1)$ & $48(28.9)$ & $20(29.0)$ & $87(42.0)$ & \\
\hline MCT4 in stroma & & & & & & $<0.001$ \\
\hline Negative & $418(56.5)$ & $222(74.5)$ & $81(48.8)$ & $23(33.3)$ & $92(44.4)$ & \\
\hline Positive & $322(43.5)$ & $76(25.5)$ & $85(51.2)$ & $46(66.7)$ & $115(55.6)$ & \\
\hline Cytoplasmic Beclin-1 & & & & & & 0.137 \\
\hline Negative & $406(54.9)$ & $169(56.7)$ & 99 (59.6) & $31(44.9)$ & $107(51.7)$ & \\
\hline Positive & $334(45.1)$ & $129(43.3)$ & $67(33.7)$ & $38(55.1)$ & $100(48.3)$ & \\
\hline Nuclear Beclin-1 & & & & & & $<0.001$ \\
\hline Negative & $666(90.0)$ & $262(87.9)$ & 152 (91.6) & $55(79.7)$ & $197(95.2)$ & \\
\hline Positive & $74(10.0)$ & $36(12.1)$ & $14(8.4)$ & $14(20.3)$ & $10(4.8)$ & \\
\hline
\end{tabular}


Table 4 Expression of metabolism-related proteins according to breast cancer phenotype ${ }^{\text {a }}$ (Continued)

\begin{tabular}{|c|c|c|c|c|c|c|}
\hline LC3A in tumor & & & & & & $<0.001$ \\
\hline Negative & $669(90.4)$ & $294(98.7)$ & $158(95.2)$ & 68 (98.6) & $149(72.0)$ & \\
\hline Positive & $71(9.6)$ & $4(1.3)$ & $8(4.8)$ & $1(1.4)$ & $58(28.0)$ & \\
\hline LC3A in stroma & & & & & & $<0.001$ \\
\hline Negative & $687(92.8)$ & 267 (89.6) & $151(91.0)$ & $62(89.9)$ & $207(100.0)$ & \\
\hline Positive & $53(7.2)$ & $31(10.4)$ & $15(9.0)$ & $7(10.1)$ & $0(0.0)$ & \\
\hline LC3B in tumor & & & & & & 0.013 \\
\hline Negative & $475(64.2)$ & $186(62.4)$ & $124(74.7)$ & $42(60.9)$ & $123(59.4)$ & \\
\hline Positive & $265(35.8)$ & $112(37.6)$ & $42(25.3)$ & $27(39.1)$ & $84(40.6)$ & \\
\hline LC3B in stroma & & & & & & 0.645 \\
\hline Negative & $688(93.0)$ & $277(93.0)$ & $151(91.0)$ & $65(94.2)$ & $195(94.2)$ & \\
\hline Positive & $52(7.0)$ & $21(7.0)$ & $15(9.0)$ & $4(5.8)$ & $12(5.8)$ & \\
\hline Cytoplasmic p62 in tumor & & & & & & $<0.001$ \\
\hline Negative & $274(37.0)$ & $131(44.0)$ & $51(30.7)$ & $15(21.7)$ & $77(37.2)$ & \\
\hline Positive & $466(63.0)$ & $167(56.0)$ & $115(69.3)$ & $54(78.3)$ & $130(62.8)$ & \\
\hline Nuclear p62 in tumor & & & & & & $<0.001$ \\
\hline Negative & $532(71.9)$ & $180(60.4)$ & $131(78.9)$ & $44(63.8)$ & $177(85.5)$ & \\
\hline Positive & $208(28.1)$ & 118 (39.6) & $35(21.1)$ & $25(36.2)$ & $30(14.5)$ & \\
\hline Nuclear p62 in stroma & & & & & & 0.876 \\
\hline Negative & $512(69.2)$ & $206(69.1)$ & $115(69.3)$ & $45(65.2)$ & $146(70.5)$ & \\
\hline Positive & $228(30.8)$ & $92(30.9)$ & $51(30.7)$ & $24(34.8)$ & $61(29.5)$ & \\
\hline
\end{tabular}

${ }^{\mathrm{a} B N I P 3, B C L 2 / a d e n o v i r u s ~ E 1 B ~ 19-k D a ~ i n t e r a c t i n g ~ p r o t e i n ~ 3 ; ~ C A I X, ~ c a r b o n i c ~ a n h y d r a s e ~ I X ; ~ G l u t-1, ~ g l u c o s e ~ t r a n s p o r t e r ~ 1 ; ~ L C 3 A, ~ m i c r o t u b u l e-a s s o c i a t e d ~ p r o t e i n ~} 1$ light chain $3 a ;$ LC3B, microtubule-associated protein 1 light chain 3 $\beta$; MCT4, monocarboxylate transporter 4; TNBC, triple-negative breast cancer.

\section{Correlation between tumor metabolic phenotype and clinicopathologic factors}

The correlation between the metabolic phenotype of breast cancer and clinicopathologic parameters is summarized in Table 6 and Figure 1. The metabolic phenotype was the Warburg type $(n=298,40.3 \%)$, the null type $(n=$ $326,44.0 \%)$, the mixed type $(n=62,8.4 \%)$ and the reverse Warburg type $(n=54,7.3 \%)$. Histologic grade was highest in the mixed type and lowest in the null type $(P<0.001)$. The mixed type had the highest percentage of ER and PR negativity, and the null type had the highest percentage of ER and PR positivity $(P<0.001)$. The Warburg type had the highest percentage of negative HER2 status $(P=$ 0.006). The Warburg and mixed types comprised the highest percentage of TNBC, and the reverse Warburg and null types comprised the highest percentage of luminal A types $(P<0.001)$. Stromal expression of ATP synthase and glutaminase was high in the reverse Warburg and mixed types and low in the Warburg and null types $(P<0.001)$. For the status of tumor autophagy, the mixed type had the highest percentage of activated tumor autophagy and the null type had the highest percentage of nonactivated tumor autophagy $(P<0.001)$. For the status of stromal autophagy, the reverse
Warburg and mixed types had a higher percentage of activation than other types $(P<0.001)$. Tumor expression of MCT4 was highest in the Warburg type and lowest in the null type $(P<0.001)$, whereas stromal expression of MCT4 was highest in the mixed type and lowest in the null type $(P<0.001)$. Ki-67 LI was highest in the mixed type and lowest in the null type $(P<0.001)$.

\section{Impact of metabolism-related proteins on patient prognosis}

The results of univariate analysis on the correlation between metabolism-related proteins and the clinicopathologic parameters of patients are summarized in Table 7. Shorter disease-free survival (DFS) was associated with Glut-1 positivity $(P=0.010)$, BNIP3 negativity, tumor phenotype (HER2 and TNBC; $P<0.001$ ) and tumor metabolic type (reverse Warburg type; $P=0.037$ ) (Figure 2). Shorter overall survival (OS) was associated with Glut-1 positivity $(P=0.023)$, tumor phenotype (HER2 and TNBC; $P<0.001$ ) and tumor metabolic type (mixed type; $P=0.045$ ) (Figure 2). Prognostic factors evaluated by multivariate Cox analysis included histologic grade, $\mathrm{T}$ stage, $\mathrm{N}$ stage, ER status, PR status, HER2 status, tumor phenotype, tumor metabolic phenotype and tumor expression of Glut-1. The results showed that the independent factors 
Table 5 Correlations between the expression of metabolism-related proteins and clinicopathologic parameters ${ }^{a}$

\begin{tabular}{|c|c|c|c|c|c|c|c|c|c|c|c|c|}
\hline \multirow[t]{3}{*}{ Parameters } & \multicolumn{3}{|c|}{ Glut-1 in tumor } & \multicolumn{3}{|c|}{ Glut-1 in stroma } & \multicolumn{3}{|c|}{ CAIX in tumor } & \multicolumn{3}{|c|}{ CAIX in stroma } \\
\hline & Negative & Positive & & Negative & Positive & & Negative & Positive & & Negative & Positive & \\
\hline & $\begin{array}{l}(n=504) \\
(\%)\end{array}$ & $\begin{array}{l}(n=236) \\
(\%)\end{array}$ & $P$-value & $\begin{array}{l}(n=724) \\
(\%)\end{array}$ & $\begin{array}{l}(n=16) \\
(\%)\end{array}$ & $P$-value & $\begin{array}{l}(n=520) \\
(\%)\end{array}$ & $\begin{array}{l}(n=220) \\
(\%)\end{array}$ & $P$-value & $\begin{array}{l}(n=627) \\
(\%)\end{array}$ & $\begin{array}{l}(n=113) \\
(\%)\end{array}$ & $P$-value \\
\hline $\begin{array}{l}\text { Age (years, } \\
\text { mean } \pm \text { SD) }\end{array}$ & $50.5 \pm 10.7$ & $48.1 \pm 11.4$ & 0.126 & $49.7 \pm 110.0$ & $49.3 \pm 9.0$ & 18.14 & $49.7 \pm 11.0$ & $49.8 \pm 11.0$ & 19.67 & $49.3 \pm 11.1$ & $51.7 \pm 10.3$ & 0.840 \\
\hline Histologic grade & & & $<0.001$ & & & 8.946 & & & 0.441 & & & 0.483 \\
\hline$|/| \mid$ & $392(71.8)$ & 99 (41.9) & & $482(67.3)$ & $9(56.3)$ & & $359(69.0)$ & $132(60.0)$ & & $427(68.1)$ & $64(56.6)$ & \\
\hline III & $112(22.2)$ & $137(58.1)$ & & $242(33.4)$ & $7(43.7)$ & & $161(31.0)$ & $88(40.0)$ & & $200(31.9)$ & $49(43.4)$ & \\
\hline ER & & & $<0.001$ & & & 0.378 & & & 0.042 & & & 1.113 \\
\hline Negative & $128(25.4)$ & $158(66.9)$ & & $275(38.0)$ & $11(68.8)$ & & $182(35.0)$ & $104(47.3)$ & & $233(37.2)$ & $53(46.9)$ & \\
\hline Positive & $376(74.6)$ & 78 (33.1) & & $449(62.0)$ & $5(31.2)$ & & $338(65.0)$ & $116(52.7)$ & & $394(62.8)$ & $60(53.1)$ & \\
\hline$P R$ & & & $<0.001$ & & & 4.305 & & & 10.92 & & & 8.715 \\
\hline Negative & $190(37.7)$ & $182(77.1)$ & & 361 (49.9) & $11(68.8)$ & & $257(49.4)$ & $115(52.3)$ & & 311 (49.6) & $61(54.0)$ & \\
\hline Positive & $314(62.3)$ & $54(22.9)$ & & $363(50.1)$ & $5(31.2)$ & & $263(50.6)$ & $105(47.7)$ & & $316(50.4)$ & $52(46.0)$ & \\
\hline HER2 & & & 0.714 & & & 6.741 & & & 0.294 & & & 1.260 \\
\hline Negative & $386(76.6)$ & $197(83.5)$ & & $572(79.0)$ & $11(68.8)$ & & $397(76.3)$ & $186(84.5)$ & & $502(80.1)$ & $81(71.7)$ & \\
\hline Positive & $118(23.4)$ & 39 (16.5) & & $152(21.0)$ & $5(31.2)$ & & $123(23.7)$ & $34(15.5)$ & & $125(19.9)$ & $32(28.3)$ & \\
\hline Tumor stage & & & $<0.001$ & & & 18.81 & & & 19.65 & & & 3.192 \\
\hline T1 & $270(53.6)$ & $88(37.3)$ & & $350(48.3)$ & $8(50.0)$ & & $251(48.3)$ & $107(48.6)$ & & $296(47.2)$ & $62(54.9)$ & \\
\hline $\mathrm{T} 2 / \mathrm{T} 3$ & $234(46.4)$ & $148(62.7)$ & & $374(51.7)$ & $8(50.0)$ & & $269(51.7)$ & $113(51.4)$ & & $331(52.8)$ & $51(45.1)$ & \\
\hline Nodal stage & & & 1.932 & & & 16.12 & & & 15.62 & & & 15.87 \\
\hline No & $286(56.7)$ & $150(63.6)$ & & $426(58.8)$ & $10(62.5)$ & & $304(58.5)$ & $132(60.0)$ & & $371(59.2)$ & $65(57.5)$ & \\
\hline N1/N2/N3 & $218(43.3)$ & $86(36.4)$ & & $298(41.2)$ & $6(37.5)$ & & $216(41.5)$ & $88(40.0)$ & & $256(40.8)$ & $48(42.5)$ & \\
\hline $\begin{array}{l}\text { Ki-67 LI (\%, } \\
\text { mean } \pm \text { SD) }\end{array}$ & $12.7 \pm 14.9$ & $29.6 \pm 22.1$ & $<0.001$ & $18.0 \pm 19.3$ & $22.5 \pm 14.7$ & 7.497 & $16.1 \pm 17.5$ & $22.7 \pm 22.1$ & $<0.001$ & $17.8 \pm 19.7$ & $19.5 \pm 16.7$ & 8.505 \\
\hline Tumor recurrence & & & 0.210 & & & 8.106 & & & 18.62 & & & 17.85 \\
\hline Absent & $467(92.7)$ & $204(86.4)$ & & $655(90.5)$ & $16(100.0)$ & & $471(90.6)$ & $200(90.9)$ & & $568(90.6)$ & $103(91.2)$ & \\
\hline Present & $37(7.3)$ & $32(13.6)$ & & $69(9.5)$ & $0(0.0)$ & & $49(9.4)$ & $20(9.1)$ & & $59(9.4)$ & $10(9.8)$ & \\
\hline Death & & & 0.420 & & & 8.085 & & & 11.76 & & & 15.22 \\
\hline Survival & $467(92.7)$ & $206(87.3)$ & & $657(90.7)$ & $16(100.0)$ & & $475(91.3)$ & $198(90.0)$ & & $571(91.1)$ & $102(90.3)$ & \\
\hline Death & $37(7.3)$ & 30 (12.7) & & $67(9.3)$ & $0(0.0)$ & & $45(8.7)$ & $22(10.0)$ & & $56(8.9)$ & $11(9.7)$ & \\
\hline
\end{tabular}


Table 5 Correlations between the expression of metabolism-related proteins and clinicopathologic parameters ${ }^{\mathrm{a}}$ (Continued)

\begin{tabular}{|c|c|c|c|c|c|c|c|c|c|c|c|c|}
\hline \multirow[t]{3}{*}{ Parameters } & \multicolumn{3}{|c|}{ ATP synthase in tumor } & \multicolumn{3}{|c|}{ ATP synthase in stroma } & \multicolumn{3}{|c|}{ Glutaminase in tumor } & \multicolumn{3}{|c|}{ Glutaminase in stroma } \\
\hline & Negative & Positive & & Negative & Positive & & Negative & Positive & & Negative & Positive & \\
\hline & $\begin{array}{l}(n=30) \\
(\%)\end{array}$ & $\begin{array}{l}(n=710) \\
(\%)\end{array}$ & $P$-value & $\begin{array}{l}(n=570) \\
(\%)\end{array}$ & $\begin{array}{l}(n=170) \\
(\%)\end{array}$ & $P$-value & $\begin{array}{l}(n=219) \\
(\%)\end{array}$ & $\begin{array}{l}(n=521) \\
(\%)\end{array}$ & $P$-value & $\begin{array}{l}(n=495) \\
(\%)\end{array}$ & $\begin{array}{l}(n=245) \\
(\%)\end{array}$ & $P$-value \\
\hline $\begin{array}{l}\text { Age (years, } \\
\text { mean } \pm \text { SD) }\end{array}$ & $46.7 \pm 9.3$ & $49.9 \pm 11.0$ & 2.478 & $49.5 \pm 11.2$ & $50.4 \pm 10.0$ & 7.539 & $49.2 \pm 11.6$ & $49.9 \pm 10.7$ & 11.90 & $49.5 \pm 11.1$ & $50.4 \pm 10.7$ & 5.124 \\
\hline Histologic grade & & & 11.63 & & & 0.252 & & & 16.77 & & & 0.168 \\
\hline$|/| \mid$ & $22(733)$ & $469(66.1)$ & & $392(68.8)$ & $99(58.2)$ & & $147(67.1)$ & $344(66.0)$ & & $345(69.7)$ & $146(59.6)$ & \\
\hline III & $8(26.7)$ & $241(33.9)$ & & $178(31.2)$ & $71(41.8)$ & & $72(32.9)$ & $177(34.0)$ & & $150(30.3)$ & $99(40.4)$ & \\
\hline ER & & & 1.827 & & & 1.533 & & & 4.536 & & & 0.126 \\
\hline Negative & $7(23.3)$ & $279(39.3)$ & & $210(36.8)$ & $76(44.7)$ & & $77(35.2)$ & $209(40.1)$ & & $174(35.2)$ & $112(45.7)$ & \\
\hline Positive & $23(76.7)$ & $431(60.7)$ & & $360(63.2)$ & $94(55.3)$ & & $142(64.8)$ & $312(59.9)$ & & $321(64.8)$ & $133(54.3)$ & \\
\hline$P R$ & & & 0.189 & & & 0.609 & & & 4.158 & & & 0.735 \\
\hline Negative & $8(26.7)$ & $364(51.3)$ & & $274(48.1)$ & $98(57.6)$ & & $102(46.6)$ & $270(51.8)$ & & $235(47.5)$ & $137(55.9)$ & \\
\hline Positive & $22(73.3)$ & 346 (48.7) & & $296(51.9)$ & $72(42.4)$ & & $117(53.4)$ & $251(48.2)$ & & $260(52.5)$ & $108(44.1)$ & \\
\hline HER2 & & & 3.570 & & & $<0.001$ & & & 13.08 & & & 3.822 \\
\hline Negative & $27(90.0)$ & $556(78.3)$ & & $472(82.8)$ & $111(65.3)$ & & $170(77.6)$ & $413(79.3)$ & & 397 (80.2) & $186(75.9)$ & \\
\hline Positive & $3(10.0)$ & $154(21.7)$ & & $98(17.2)$ & $59(34.7)$ & & $49(22.4)$ & $108(20.7)$ & & 98 (19.8) & $59(24.1)$ & \\
\hline Tumor stage & & & 7.539 & & & 21.00 & & & 7.875 & & & 18.39 \\
\hline $\mathrm{T} 1$ & $12(40.0)$ & $346(48.7)$ & & $276(48.4)$ & $82(48.2)$ & & $100(45.7)$ & $258(49.5)$ & & $238(48.1)$ & $120(49.0)$ & \\
\hline $\mathrm{T} 2 / \mathrm{T} 3$ & $18(60.0)$ & $364(51.3)$ & & $294(51.6)$ & $88(51.8)$ & & $119(54.3)$ & $263(50.5)$ & & $257(51.9)$ & $125(51.0)$ & \\
\hline Nodal stage & & & 7.266 & & & 6.027 & & & 7.728 & & & 8.967 \\
\hline No & $15(50.0)$ & $421(59.3)$ & & $342(60.0)$ & $94(55.3)$ & & $135(61.6)$ & 301 (57.8) & & $297(60.0)$ & $139(56.7)$ & \\
\hline N1/N2/N3 & $15(50.0)$ & $289(40.7)$ & & $228(40.0)$ & $76(44.7)$ & & $84(38.4)$ & $220(42.2)$ & & $198(40.0)$ & $106(43.3)$ & \\
\hline $\begin{array}{l}\mathrm{Ki}-67 \mathrm{LI}(\%, \\
\text { mean } \pm \text { SD) }\end{array}$ & $9.8 \pm 11.7$ & $18.4 \pm 19.4$ & 0.336 & $17.3 \pm 19.7$ & $20.8 \pm 17.5$ & 0.798 & $18.6 \pm 20.0$ & $17.8 \pm 18.9$ & 13.50 & $15.6 \pm 17.2$ & $22.9 \pm 22.0$ & 0.021 \\
\hline Tumor recurrence & & & 10.79 & & & 21.00 & & & 7.014 & & & 10.56 \\
\hline Absent & $26(86.7)$ & 645 (90.8) & & $517(90.7)$ & $154(90.6)$ & & $195(89.0)$ & $476(91.4)$ & & $446(90.1)$ & $225(91.8)$ & \\
\hline Present & $4(13.3)$ & $65(9.2)$ & & $53(9.3)$ & $16(9.4)$ & & $24(11.0)$ & $45(8.6)$ & & $49(9.9)$ & $20(8.2)$ & \\
\hline Death & & & 15.68 & & & 13.60 & & & 3.381 & & & 8.757 \\
\hline Survival & 27 (90.0) & $646(91.0)$ & & $520(91.2)$ & $153(90.0)$ & & $194(88.6)$ & 479 (91.9) & & 447 (90.3) & $226(92.2)$ & \\
\hline Death & $3(10.0)$ & $64(9.0)$ & & $50(8.8)$ & $17(10.0)$ & & 25 (11.4) & $42(8.1)$ & & $48(9.7)$ & $19(7.8)$ & \\
\hline
\end{tabular}


Table 5 Correlations between the expression of metabolism-related proteins and clinicopathologic parameters ${ }^{\mathrm{a}}$ (Continued)

\begin{tabular}{|c|c|c|c|c|c|c|c|c|c|c|c|c|}
\hline \multirow[t]{3}{*}{ Parameters } & \multicolumn{3}{|c|}{ BNIP3 in tumor } & \multicolumn{3}{|c|}{ BNIP3 in stroma } & \multicolumn{3}{|c|}{ MCT4 in tumor } & \multicolumn{3}{|c|}{ MCT4 in stroma } \\
\hline & Negative & Positive & & Negative & Positive & & Negative & Positive & & Negative & Positive & \\
\hline & $\begin{array}{l}(n=504) \\
(\%)\end{array}$ & $\begin{array}{l}(n=236) \\
(\%)\end{array}$ & $P$-value & $\begin{array}{l}(n=700) \\
(\%)\end{array}$ & $\begin{array}{l}(n=40) \\
(\%)\end{array}$ & $P$-value & $\begin{array}{l}(n=540) \\
(\%)\end{array}$ & $\begin{array}{l}(n=200) \\
(\%)\end{array}$ & $P$-value & $\begin{array}{l}(n=418) \\
(\%)\end{array}$ & $\begin{array}{l}(n=322) \\
(\%)\end{array}$ & $P$-value \\
\hline $\begin{array}{l}\text { Age } \\
\text { (years, mean } \pm S D)\end{array}$ & $48.9 \pm 10.9$ & $51.6 \pm 11.0$ & 0.042 & $49.5 \pm 10.8$ & $53.2 \pm 12.7$ & 0.882 & $49.8 \pm 11.1$ & $49.6 \pm 10.6$ & 17.91 & $49.6 \pm 11.0$ & $49.5 \pm 10.9$ & 14.80 \\
\hline Histologic grade & & & 5.859 & & & 15.37 & & & $<0.001$ & & & $<0.001$ \\
\hline$|/| \mid$ & $341(67.7)$ & $150(63.6)$ & & $463(66.1)$ & $28(70.0)$ & & $386(71.5)$ & $105(52.5)$ & & $314(75.1)$ & $177(55.0)$ & \\
\hline III & $163(32.3)$ & $86(36.4)$ & & 237 (33.9) & $12(30.0)$ & & $154(28.5)$ & 95 (47.5) & & $104(24.9)$ & $145(45.0)$ & \\
\hline ER & & & 14.40 & & & 12.99 & & & $<0.001$ & & & $<0.001$ \\
\hline Negative & $192(38.1)$ & $94(39.8)$ & & $269(38.4)$ & $17(42.5)$ & & $174(32.2)$ & $112(56.0)$ & & $123(29.4)$ & $163(50.6)$ & \\
\hline Positive & $312(61.9)$ & $142(60.2)$ & & $431(61.6)$ & $23(57.5)$ & & $366(67.8)$ & $88(44.0)$ & & $295(70.6)$ & $159(49.4)$ & \\
\hline$P R$ & & & 17.07 & & & 8.757 & & & $<0.001$ & & & $<0.001$ \\
\hline Negative & $255(50.6)$ & $117(49.6)$ & & $349(49.9)$ & $23(57.5)$ & & $232(43.0)$ & $140(70.0)$ & & $179(42.8)$ & $193(60.0)$ & \\
\hline Positive & $249(49.4)$ & $119(50.4)$ & & $351(50.1)$ & $17(42.5)$ & & $308(57.0)$ & $60(30.0)$ & & $239(57.2)$ & $129(40.0)$ & \\
\hline HER2 & & & 4.410 & & & 6.762 & & & 11.42 & & & $<0.001$ \\
\hline Negative & $404(80.2)$ & 179 (75.8) & & $554(79.1)$ & $29(72.5)$ & & $422(78.1)$ & $161(80.5)$ & & $356(85.2)$ & $227(70.5)$ & \\
\hline Positive & $100(19.8)$ & $57(24.2)$ & & $146(20.9)$ & $11(27.5)$ & & $118(21.9)$ & 39 (19.5) & & $62(14.8)$ & $95(29.5)$ & \\
\hline Tumor stage & & & 1.449 & & & 15.66 & & & $<0.001$ & & & 7.854 \\
\hline $\mathrm{T} 1$ & $232(46.0)$ & $126(53.4)$ & & $340(48.6)$ & $18(45.0)$ & & $283(52.4)$ & 75 (37.5) & & $196(46.9)$ & $162(50.3)$ & \\
\hline $\mathrm{T} 2 / \mathrm{T} 3$ & $272(54.0)$ & $110(46.6)$ & & $360(51.4)$ & $22(55.0)$ & & $257(47.6)$ & $125(62.5)$ & & $222(53.1)$ & $160(49.7)$ & \\
\hline Nodal stage & & & 6.237 & & & 0.630 & & & 18.20 & & & 7.686 \\
\hline NO & $290(57.5)$ & $146(61.9)$ & & $419(59.9)$ & $17(42.5)$ & & $317(58.7)$ & $119(59.5)$ & & $240(57.4)$ & $196(60.9)$ & \\
\hline $\mathrm{N} 1 / \mathrm{N} 2 / \mathrm{N} 3$ & $214(42.5)$ & $90(38.1)$ & & $281(40.1)$ & $23(57.5)$ & & $223(41.3)$ & $81(40.5)$ & & $178(42.6)$ & $126(39.1)$ & \\
\hline $\begin{array}{l}\text { Ki-67 LI (\%, } \\
\text { mean } \pm \text { SD) }\end{array}$ & $18.9 \pm 20.9$ & $16.2 \pm 15.0$ & 1.680 & $18.3 \pm 19.5$ & $14.6 \pm 13.7$ & 5.208 & $15.3 \pm 17.8$ & $25.6 \pm 21.0$ & $<0.001$ & $13.2 \pm 16.5$ & $24.3 \pm 20.7$ & $<0.001$ \\
\hline Tumor recurrence & & & 0.021 & & & 3.444 & & & 14.11 & & & 11.00 \\
\hline Absent & 445 (88.3) & $226(95.8)$ & & $632(90.3)$ & 39 (97.5) & & $491(90.9)$ & $180(90.0)$ & & $376(90.0)$ & 295 (91.6) & \\
\hline Present & $59(11.7)$ & $10(4.2)$ & & $68(9.7)$ & $1(2.5)$ & & $49(9.1)$ & $20(10.0)$ & & $42(10.0)$ & $27(8.4)$ & \\
\hline Death & & & 5.712 & & & 15.22 & & & 11.90 & & & 2.562 \\
\hline Survival & $454(90.1)$ & $219(92.8)$ & & $636(90.9)$ & $37(92.5)$ & & $493(91.3)$ & $180(90.0)$ & & $374(89.5)$ & $299(92.9)$ & \\
\hline Death & $50(9.9)$ & $17(7.2)$ & & $64(9.1)$ & $3(7.5)$ & & $47(8.7)$ & $20(10.0)$ & & $44(10.5)$ & $23(7.1)$ & \\
\hline
\end{tabular}


Table 5 Correlations between the expression of metabolism-related proteins and clinicopathologic parameters ${ }^{\mathrm{a}}$ (Continued)

\begin{tabular}{|c|c|c|c|c|c|c|c|c|c|c|c|c|}
\hline \multirow[t]{3}{*}{ Parameters } & \multicolumn{3}{|c|}{ Cytoplasmic Beclin-1 } & \multicolumn{3}{|c|}{ Nuclear Beclin-1 } & \multicolumn{3}{|c|}{ LC3A in tumor } & \multicolumn{3}{|c|}{ LC3A in stroma } \\
\hline & Negative & Positive & & Negative & Positive & & Negative & Positive & & Negative & Positive & \\
\hline & $\begin{array}{l}(n=406) \\
(\%)\end{array}$ & $\begin{array}{l}(n=334) \\
(\%)\end{array}$ & $P$-value & $\begin{array}{l}(n=666) \\
(\%)\end{array}$ & $\begin{array}{l}(n=74) \\
(\%)\end{array}$ & $P$-value & $\begin{array}{l}(n=669) \\
(\%)\end{array}$ & $\begin{array}{l}(n=71) \\
(\%)\end{array}$ & $P$-value & $\begin{array}{l}(n=687) \\
(\%)\end{array}$ & $\begin{array}{l}(n=53) \\
(\%)\end{array}$ & $P$-value \\
\hline $\begin{array}{l}\text { Age (years, } \\
\text { mean } \pm \text { SD) }\end{array}$ & $48.6 \pm 10.5$ & $51.1 \pm 11.4$ & 0.042 & $49.6 \pm 11.1$ & $50.8 \pm 9.9$ & 8.316 & $50.2 \pm 11.0$ & $45.6 \pm 9.8$ & 0.021 & $49.7 \pm 11.0$ & $49.6 \pm 9.2$ & 19.65 \\
\hline Histologic grade & & & 14.61 & & & $<0.001$ & & & $<0.001$ & & & 1.029 \\
\hline$|/| \mid$ & $272(67.0)$ & $219(65.6)$ & & $427(64.1)$ & $64(86.5)$ & & $470(70.3)$ & $21(29.6)$ & & $449(65.4)$ & $42(79.2)$ & \\
\hline III & $134(33.0)$ & $115(34.4)$ & & $239(35.9)$ & $10(13.5)$ & & $199(29.7)$ & $50(70.4)$ & & $238(34.6)$ & $11(20.8)$ & \\
\hline ER & & & 6.090 & & & 8.022 & & & $<0.001$ & & & $<0.001$ \\
\hline Negative & $143(35.2)$ & $143(42.8)$ & & $261(39.2)$ & 25 (33.8) & & $226(33.8)$ & $60(84.5)$ & & $278(40.5)$ & $8(15.1)$ & \\
\hline Positive & $263(64.8)$ & $191(57.2)$ & & $405(60.8)$ & $49(66.2)$ & & $443(66.2)$ & $11(15.5)$ & & $409(59.5)$ & $45(84.9)$ & \\
\hline$P R$ & & & 19.76 & & & 1.827 & & & $<0.001$ & & & $<0.001$ \\
\hline Negative & $205(50.5)$ & $167(50.0)$ & & $342(51.4)$ & $30(40.5)$ & & $309(46.2)$ & $63(88.7)$ & & $360(52.4)$ & $12(22.6)$ & \\
\hline Positive & $201(49.5)$ & $167(50.0)$ & & $324(48.6)$ & $44(59.5)$ & & $360(53.8)$ & $8(11.3)$ & & $327(47.6)$ & $41(77.4)$ & \\
\hline HER2 & & & 12.36 & & & 0.336 & & & $<0.001$ & & & 8.022 \\
\hline Negative & $323(79.6)$ & $260(77.8)$ & & $533(80.0)$ & $50(67.6)$ & & $515(76.9)$ & $68(95.8)$ & & $544(79.2)$ & $39(73.6)$ & \\
\hline Positive & $83(20.4)$ & $74(22.2)$ & & $133(20.0)$ & $24(32.4)$ & & $154(23.1)$ & $3(4.2)$ & & $143(20.8)$ & $14(26.4)$ & \\
\hline Tumor stage & & & 0.042 & & & 6.888 & & & 16.86 & & & 0.210 \\
\hline $\mathrm{T} 1$ & $175(43.1)$ & $183(54.8)$ & & $318(47.7)$ & $40(54.1)$ & & $325(48.6)$ & $33(46.5)$ & & $323(47.0)$ & $35(66.0)$ & \\
\hline $\mathrm{T} 2 / \mathrm{T} 3$ & $231(56.9)$ & $151(45.2)$ & & $348(52.3)$ & $34(45.9)$ & & $344(51.4)$ & $38(53.5)$ & & $364(53.0)$ & $18(34.0)$ & \\
\hline Nodal stage & & & 13.71 & & & 3.612 & & & 6.552 & & & 16.23 \\
\hline NO & $236(58.1)$ & $200(59.9)$ & & $398(59.8)$ & $38(51.4)$ & & $390(58.3)$ & $46(64.8)$ & & $406(59.1)$ & $30(56.6)$ & \\
\hline N1/N2/N3 & $170(41.9)$ & $134(40.1)$ & & $268(40.2)$ & $36(48.6)$ & & $279(41.7)$ & $25(35.2)$ & & $281(40.9)$ & $23(43.4)$ & \\
\hline $\begin{array}{l}\text { Ki-67 LI (\%, } \\
\text { mean } \pm \text { SD) }\end{array}$ & $17.8 \pm 19.4$ & $18.3 \pm 19.1$ & 0.042 & $19.0 \pm 19.8$ & $9.5 \pm 10.0$ & $<0.001$ & $15.7 \pm 17.2$ & $39.6 \pm 23.1$ & $<0.001$ & $18.7 \pm 19.7$ & $10.4 \pm 9.6$ & 0.032 \\
\hline Tumor recurrence & & & 0.882 & & & 2.877 & & & 4.053 & & & 16.98 \\
\hline Absent & $360(88.7)$ & $311(93.1)$ & & $600(90.1)$ & $71(95.9)$ & & $610(91.2)$ & $61(85.9)$ & & $622(90.5)$ & $49(92.5)$ & \\
\hline Present & $46(11.3)$ & $23(6.9)$ & & $66(9.9)$ & $3(4.1)$ & & $59(8.8)$ & $10(14.1)$ & & $65(9.5)$ & $4(7.5)$ & \\
\hline Death & & & 16.75 & & & 0.189 & & & 10.75 & & & 9.765 \\
\hline Survival & 368 (90.6) & $305(91.3)$ & & $600(90.1)$ & $73(98.6)$ & & $610(91.2)$ & $63(88.7)$ & & $623(90.7)$ & $50(94.3)$ & \\
\hline Death & $38(9.4)$ & $29(8.7)$ & & $66(9.9)$ & $1(1.4)$ & & $59(8.8)$ & $8(11.3)$ & & $64(9.3)$ & $3(5.7)$ & \\
\hline
\end{tabular}


Table 5 Correlations between the expression of metabolism-related proteins and clinicopathologic parameters ${ }^{\mathrm{a}}$ (Continued)

\begin{tabular}{|c|c|c|c|c|c|c|c|c|c|c|c|c|c|c|c|}
\hline \multirow[t]{3}{*}{ Parameters } & \multicolumn{3}{|c|}{ LC3B in tumor } & \multicolumn{3}{|c|}{ LC3B in stroma } & \multicolumn{3}{|c|}{ Cytoplasmic p62 in tumor } & \multicolumn{3}{|c|}{ Nuclear p62 in tumor } & \multicolumn{3}{|c|}{ Nuclear p62 in stroma } \\
\hline & Negative & Positive & & Negative & Positive & & Negative & Positive & & Negative & Positive & & Negative & Positive & \\
\hline & $\begin{array}{l}(n=475) \\
(\%)\end{array}$ & $\begin{array}{l}(n=265) \\
(\%)\end{array}$ & $P$-value & $\begin{array}{l}(n=688) \\
(\%)\end{array}$ & $\begin{array}{l}(n=52) \\
(\%)\end{array}$ & $P$-value & $\begin{array}{l}(n=274) \\
(\%)\end{array}$ & $\begin{array}{l}(n=466) \\
(\%)\end{array}$ & $P$-value & $\begin{array}{l}(n=532) \\
(\%)\end{array}$ & $\begin{array}{l}(n=208) \\
(\%)\end{array}$ & $P$-value & $\begin{array}{l}(n=512) \\
(\%)\end{array}$ & $\begin{array}{l}(n=228) \\
(\%)\end{array}$ & $P$-value \\
\hline Age & $49.4 \pm 10.4$ & $50.4 \pm 12.0$ & 4.599 & $49.6 \pm 11.0$ & $51.1 \pm 10.6$ & 15.77 & $49.4 \pm 10.3$ & $49.9 \pm 11.4$ & 10.35 & $49.4 \pm 10.9$ & $50.6 \pm 11.2$ & 3.717 & $49.5 \pm 11.2$ & $50.3 \pm 10.4$ & 6.741 \\
\hline \multicolumn{16}{|l|}{ (years, mean $\pm S D$ ) } \\
\hline Histologic grade & & & 3.528 & & & 9.450 & & & 0.210 & & & $<0.001$ & & & 18.18 \\
\hline$|/| \mid$ & $324(68.2)$ & $167(63.0)$ & & $459(66.7)$ & $32(61.5)$ & & $198(72.3)$ & $293(62.9)$ & & $322(60.5)$ & $169(81.3)$ & & $341(66.6)$ & $150(65.8)$ & \\
\hline III & $151(31.8)$ & $98(37.0)$ & & $229(33.3)$ & $20(38.5)$ & & $76(27.7)$ & $173(37.1)$ & & $210(39.5)$ & $39(18.8)$ & & $171(33.4)$ & $78(34.2)$ & \\
\hline ER & & & 6.279 & & & 7.980 & & & 2.877 & & & $<0.001$ & & & 14.34 \\
\hline Negative & $173(36.4)$ & $113(42.6)$ & & $269(39.1)$ & $17(32.7)$ & & $96(35.0)$ & $190(40.8)$ & & $227(42.7)$ & $59(28.4)$ & & $195(38.1)$ & 91 (39.9) & \\
\hline Positive & 302 (63.6) & $152(57.4)$ & & $419(60.9)$ & $35(67.3)$ & & $178(65.0)$ & $276(59.2)$ & & $305(57.3)$ & 149 (71.6) & & $317(61.9)$ & $137(60.1)$ & \\
\hline PR & & & 10.29 & & & 5.292 & & & 0.105 & & & $<0.001$ & & & 17.05 \\
\hline Negative & $234(49.3)$ & $138(52.1)$ & & $350(50.9)$ & $22(42.3)$ & & $119(43.4)$ & $253(54.3)$ & & $293(55.1)$ & 79 (38.0) & & $259(50.6)$ & $113(49.6)$ & \\
\hline Positive & $241(50.7)$ & $127(47.9)$ & & $338(49.1)$ & $30(57.7)$ & & $155(56.6)$ & $213(45.7)$ & & $239(44.9)$ & $129(62.0)$ & & $253(49.4)$ & 115 (50.4) & \\
\hline HER2 & & & 12.07 & & & 12.55 & & & $<0.001$ & & & 16.04 & & & 13.16 \\
\hline Negative & 371 (78.1) & $212(80.0)$ & & $540(78.5)$ & $43(82.7)$ & & $238(86.9)$ & $345(74.0)$ & & $421(79.1)$ & $162(77.9)$ & & $406(79.3)$ & $177(34.6)$ & \\
\hline Positive & $104(21.9)$ & $53(20.0)$ & & $148(21.5)$ & $9(17.3)$ & & $36(13.1)$ & $121(26.0)$ & & $111(20.9)$ & $46(22.1)$ & & $106(20.7)$ & $51(22.4)$ & \\
\hline Tumor stage & & & 0.357 & & & 14.00 & & & 3.570 & & & 0.189 & & & 0.189 \\
\hline $\mathrm{T} 1$ & $214(45.1)$ & $144(54.3)$ & & $331(48.1)$ & 27 (51.9) & & $142(51.8)$ & $216(46.4)$ & & $241(45.3)$ & $117(56.2)$ & & $231(45.1)$ & $127(55.7)$ & \\
\hline $\mathrm{T} 2 / \mathrm{T} 3$ & $261(54.9)$ & $121(45.7)$ & & 357 (51.9) & 25 (48.1) & & $132(48.2)$ & 250 (53.6) & & $291(54.7)$ & 91 (43.8) & & $281(54.9)$ & $101(44.3)$ & \\
\hline Nodal stage & & & 6.531 & & & 13.90 & & & 3.969 & & & 5.901 & & & 10.87 \\
\hline NO & $273(57.5)$ & $163(61.5)$ & & 407 (59.2) & 29 (55.8) & & $170(62.0)$ & $266(57.1)$ & & $320(60.2)$ & $116(55.8)$ & & $306(59.8)$ & $130(57.0)$ & \\
\hline $\mathrm{N} 1 / \mathrm{N} 2 / \mathrm{N} 3$ & $202(42.5)$ & $102(38.5)$ & & $281(40.8)$ & $23(44.2)$ & & $104(38.0)$ & $200(42.9)$ & & $212(39.8)$ & $92(44.2)$ & & $206(40.2)$ & $98(43.0)$ & \\
\hline $\begin{array}{l}\text { Ki-67 LI (\%, } \\
\text { mean } \pm \text { SD) }\end{array}$ & $18.2 \pm 19.8$ & $17.8 \pm 18.2$ & 16.10 & $18.4 \pm 19.4$ & $18.9 \pm 17.6$ & 15.77 & $16.0 \pm 19.2$ & $19.3 \pm 19.2$ & 0567 & $21.4 \pm 20.8$ & $9.5 \pm 10.6$ & $<0.001$ & $18.5 \pm 19.7$ & $17.0 \pm 18.1$ & 7.161 \\
\hline Tumor recurrence & & & 18.81 & & & 9.765 & & & 18.83 & & & 3.339 & & & 19.80 \\
\hline Absent & $430(90.5)$ & $241(90.9)$ & & $622(90.4)$ & 49 (94.2) & & $248(90.5)$ & $423(90.8)$ & & $477(89.7)$ & $194(93.3)$ & & 464 (90.6) & $207(90.8)$ & \\
\hline Present & $45(9.5)$ & $24(9.1)$ & & $66(9.6)$ & $3(5.8)$ & & $26(9.5)$ & $43(9.2)$ & & $55(10.3)$ & $14(6.7)$ & & $48(9.4)$ & $21(9.2)$ & \\
\hline Death & & & 8.925 & & & 12.89 & & & 14.53 & & & 10.03 & & & 14.28 \\
\hline Survival & 435 (91.6) & $238(89.8)$ & & $624(90.7)$ & 49 (94.2) & & 251 (91.6) & $422(90.6)$ & & $481(90.4)$ & $192(92.3)$ & & $467(91.2)$ & $206(90.3)$ & \\
\hline Death & $40(8.4)$ & $27(10.2)$ & & $64(9.3)$ & $3(5.8)$ & & $23(8.4)$ & $44(9.4)$ & & $51(9.6)$ & $16(7.7)$ & & $45(8.8)$ & $22(9.7)$ & \\
\hline
\end{tabular}

${ }^{a}$ P-values are corrected for multiple testing using the Bonferroni correction. BNIP3, BCL2/adenovirus E1B 19-kDa interacting protein 3; CAIX, carbonic anhydrase IX; Glut-1, glucose transporter 1; LC3A, microtubule-associated protein 1 light chain 3a; LC3B, microtubule-associated protein 1 light chain 3ß; LI, labeling index; MCT4, monocarboxylate transporter 4; TNBC, triple-negative breast cancer. 
Table 6 Clinicopathologic characteristics of patients according to metabolic phenotype ${ }^{a}$

\begin{tabular}{|c|c|c|c|c|c|}
\hline Parameters & $\begin{array}{l}\text { Warburg type } \\
(n=298)(\%)\end{array}$ & $\begin{array}{l}\text { Reverse Warburg } \\
\text { type }(n=54)(\%)\end{array}$ & $\begin{array}{l}\text { Mixed type } \\
(n=62)(\%)\end{array}$ & $\begin{array}{l}\text { Null type } \\
(n=326)(\%)\end{array}$ & $P$-value \\
\hline Age (years, mean $\pm S D$ ) & $48.5 \pm 11.7$ & $52.0 \pm 10.2$ & $51.3 \pm 10.2$ & $50.1 \pm 10.5$ & 0.052 \\
\hline Histologic grade & & & & & $<0.001$ \\
\hline$|/| \mid$ & $169(56.7)$ & $41(75.9)$ & $23(37.0)$ & $258(79.1)$ & \\
\hline III & $129(43.3)$ & $13(24.1)$ & $39(72.2)$ & $68(20.9)$ & \\
\hline Tumor stage & & & & & 0.017 \\
\hline $\mathrm{T} 1$ & $123(41.3)$ & $29(53.7)$ & $34(54.8)$ & $172(52.8)$ & \\
\hline $\mathrm{T} 2 / \mathrm{T} 3$ & $175(58.7)$ & $25(46.3)$ & $28(45.2)$ & $154(47.2)$ & \\
\hline Nodal stage & & & & & 0.457 \\
\hline NO & $177(59.3)$ & $27(50.0)$ & $40(64.5)$ & $192(58.9)$ & \\
\hline $\mathrm{N} 1 / \mathrm{N} 2 / \mathrm{N} 3$ & $121(40.6)$ & $27(50.0)$ & $22(35.5)$ & $134(41.1)$ & \\
\hline Estrogen receptor status & & & & & $<0.001$ \\
\hline Negative & $152(51.0)$ & $15(27.8)$ & $39(62.9)$ & $80(24.5)$ & \\
\hline Positive & $146(49.0)$ & $39(72.2)$ & $23(37.1)$ & $246(75.5)$ & \\
\hline Progesterone receptor status & & & & & $<0.001$ \\
\hline Negative & $181(60.7)$ & $22(40.7)$ & $40(64.5)$ & 129 (39.6) & \\
\hline Positive & $117(39.3)$ & $32(59.3)$ & $22(35.5)$ & $197(60.4)$ & \\
\hline HER2 status & & & & & 0.006 \\
\hline Negative & $252(84.6)$ & $36(66.7)$ & $47(75.8)$ & $248(76.1)$ & \\
\hline Positive & $46(15.4)$ & $18(33.3)$ & $15(24.2)$ & $78(23.9)$ & \\
\hline Molecular subtype & & & & & $<0.001$ \\
\hline Luminal A & $91(30.5)$ & $22(40.7)$ & $12(19.4)$ & $173(53.1)$ & \\
\hline Luminal B & $58(19.5)$ & $18(33.3)$ & $13(21.0)$ & $77(23.6)$ & \\
\hline HER2 & $22(7.4)$ & $7(13.0)$ & $10(16.1)$ & $30(9.2)$ & \\
\hline Triple-negative & $127(42.6)$ & $7(13.0)$ & $27(43.5)$ & $46(14.1)$ & \\
\hline ATP synthase in tumor & & & & & 0.178 \\
\hline Negative & $8(2.7)$ & $1(1.9)$ & $2(3.2)$ & $19(5.8)$ & \\
\hline Positive & $290(97.3)$ & $53(98.1)$ & $60(96.8)$ & $307(94.2)$ & \\
\hline ATP synthase in stroma & & & & & $<0.001$ \\
\hline Negative & $247(82.9)$ & $29(53.7)$ & $33(53.2)$ & $261(80.1)$ & \\
\hline Positive & $51(17.1)$ & $25(46.3)$ & $29(46.8)$ & $65(19.9)$ & \\
\hline Glutaminase in tumor & & & & & 0.512 \\
\hline Negative & $84(28.2)$ & $13(24.1)$ & $17(27.4)$ & $105(32.2)$ & \\
\hline Positive & $214(71.8)$ & $41(75.9)$ & $45(72.6)$ & $221(67.8)$ & \\
\hline Glutaminase in stroma & & & & & $<0.001$ \\
\hline Negative & $206(69.1)$ & $20(37.0)$ & $29(46.8)$ & $240(73.6)$ & \\
\hline Positive & $92(30.9)$ & $34(63.0)$ & $33(53.2)$ & $86(26.4)$ & \\
\hline Tumor mitochondrial status & & & & & 0.217 \\
\hline Dysfunctional & $94(31.5)$ & $20(37.0)$ & $26(41.9)$ & $96(29.4)$ & \\
\hline Functional & $204(68.5)$ & $34(63.0)$ & $36(58.1)$ & $230(70.6)$ & \\
\hline Stroma mitochondrial status & & & & & 0.055 \\
\hline Dysfunctional & $13(4.4)$ & $3(5.6)$ & $8(12.9)$ & $16(4.9)$ & \\
\hline Functional & $285(95.6)$ & $51(94.4)$ & $54(87.1)$ & $310(95.1)$ & \\
\hline
\end{tabular}


Table 6 Clinicopathologic characteristics of patients according to metabolic phenotype ${ }^{a}$ (Continued)

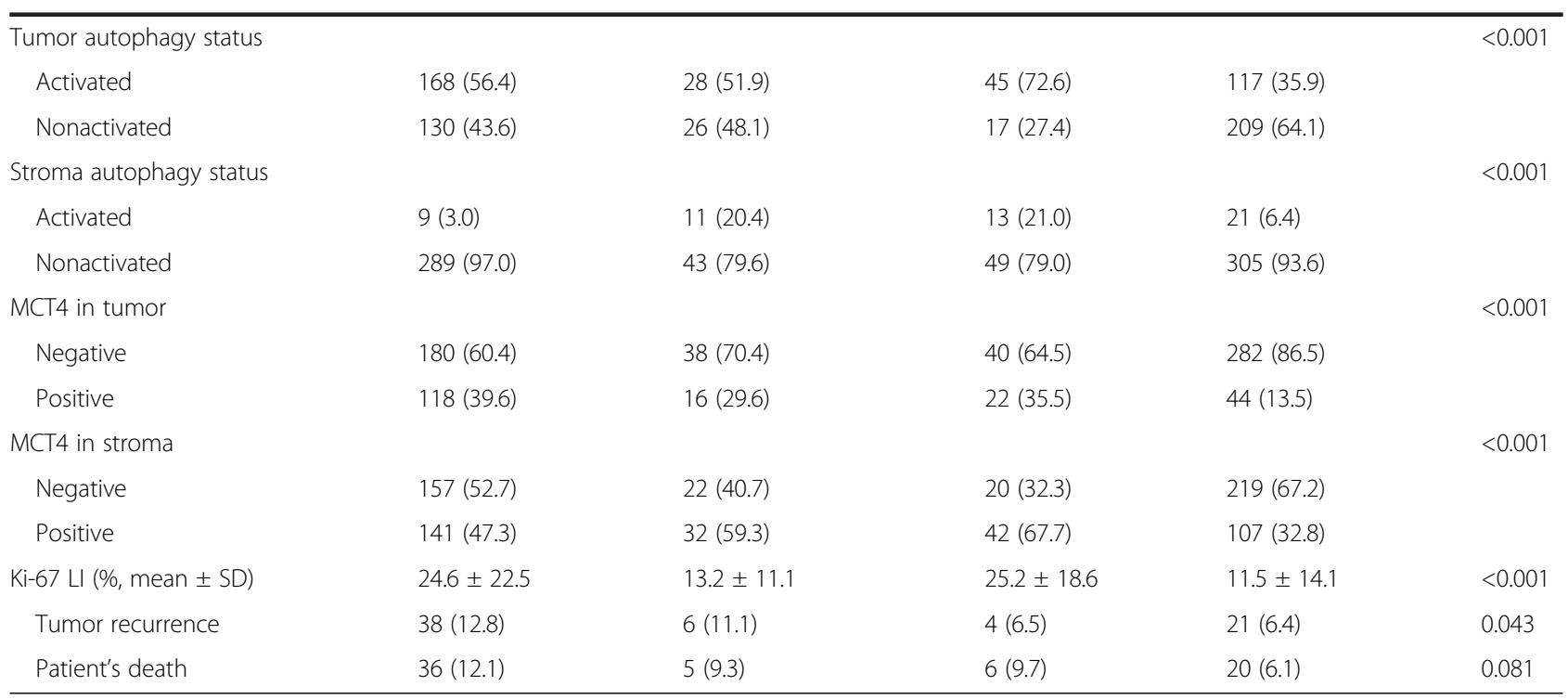

aL, labeling index; MCT4, monocarboxylate transporter 4.

associated with shorter DFS were ER negativity (odds ratio $(\mathrm{OR})=2.7,95 \% \mathrm{CI}=1.7$ to $4.5 ; P<0.001)$, $\mathrm{N}$ stage (N0 vs. $\mathrm{N} 1 / 2 / 3, \mathrm{OR}=2.3,95 \% \mathrm{CI}=1.4$ to $3.8 ; P=0.001)$ and $\mathrm{T}$ stage (T1 vs. T2/3, OR $=2.4,95 \% \mathrm{CI}=1.3$ to $4.4 ; P=0.002$ ) and those associated with shorter OS were ER negativity $(\mathrm{OR}=3.3,95 \% \mathrm{CI}=2.0$ to $5.5 ; P<0.001)$ and $\mathrm{N}$ stage (N0 vs. $\mathrm{N} 1 / 2 / 3, \mathrm{OR}=2.3,95 \% \mathrm{CI}=1.4$ to $3.8 ; P=0.001$ ).

\section{Western blot analysis of metabolism-related proteins in tumor and stroma according to tumor phenotype}

Western blot analysis was performed to investigate expression of metabolism-related proteins in tumor and stroma according to the tumor phenotype. The expression of Glut-1 and ATP synthase was higher in HER2 and TNBC types, and the expression was higher in tumor than in stroma (Figure 3). The expression of p62 was higher in tumor than stroma, regardless of the tumor phenotype.

\section{Discussion}

In the present study, we investigated the differential expression of metabolism-related markers according to the subtypes of breast cancer. Expression of glycolysis markers such as Glut-1, CAIX and MCT-4 was highest in TNBC, which is consistent with the results of previous studies showing higher expression of Glut-1 and CAIX in basal-like breast cancer [19]. The active metabolic status of a tumor can be inferred from common histologic features of TNBC, such as high-grade nuclei, highgrade prominent necrosis and increased mitotic activity [20], and this was supported by the results of IHC in the present study. Tumor expression of Glut-1 was associated with higher histologic grade $(P<0.001)$, ER negativity $(P<0.001)$, higher $\mathrm{T}$ stage $(P<0.001)$, and higher
Ki-67 LI $(P<0.001)$, whereas CAIX was associated with higher Ki-67 LI $(P<0.001)$. Tumor expression of MCT4 was associated with higher histologic grade $(P<0.001)$, ER negativity $(P<0.001)$, PR negativity $(P<0.001)$, higher $\mathrm{T}$ stage $(P<0.001)$ and higher Ki-67 LI $(P<0.001)$. In addition, expression of Glut-1, CAIX and MCT-4 was associated with factors reflecting higher metabolic status. In contrast, tumor expression of Glut-1, CAIX and MCT-4 was lowest in luminal A and B. Luminal type tumors tend to show a lower grade, lower mitotic index and less necrosis than the HER2 type or TNBC, suggesting nonactive metabolic status of the tumor, which was supported by the results of $\mathrm{IHC}$.

The expression of Glut-1, CAIX, BNIP3, MCT4, LC3A, LC3B and p62 was observed not only in tumor cells but also in stromal cells, which has not been thoroughly described in previous studies. The reverse Warburg effect theory suggests that tumor stroma, along with the tumor per se, plays a role in cancer metabolism [2-5]. According to this theory, metabolism in stromal cells occurs through glycolysis due to dysfunctional mitochondria caused by increased autophagy, whereas metabolism of tumor cells occurs through OXPHOS in functional mitochondria. This contrasts with the conventional Warburg effect theory, which states that glycolysis is the major metabolic process in tumor cells. The major metabolic phenotypes in this study were the Warburg type (40.3\%) and the null type $(44.0 \%)$, according to the metabolic processes of tumor and stromal cells. We found that each metabolic phenotype investigated had different characteristics. The mixed type had higher histologic grade, ER negativity, PR negativity and higher Ki-67 LI, in contrast to the null type, which had lower 


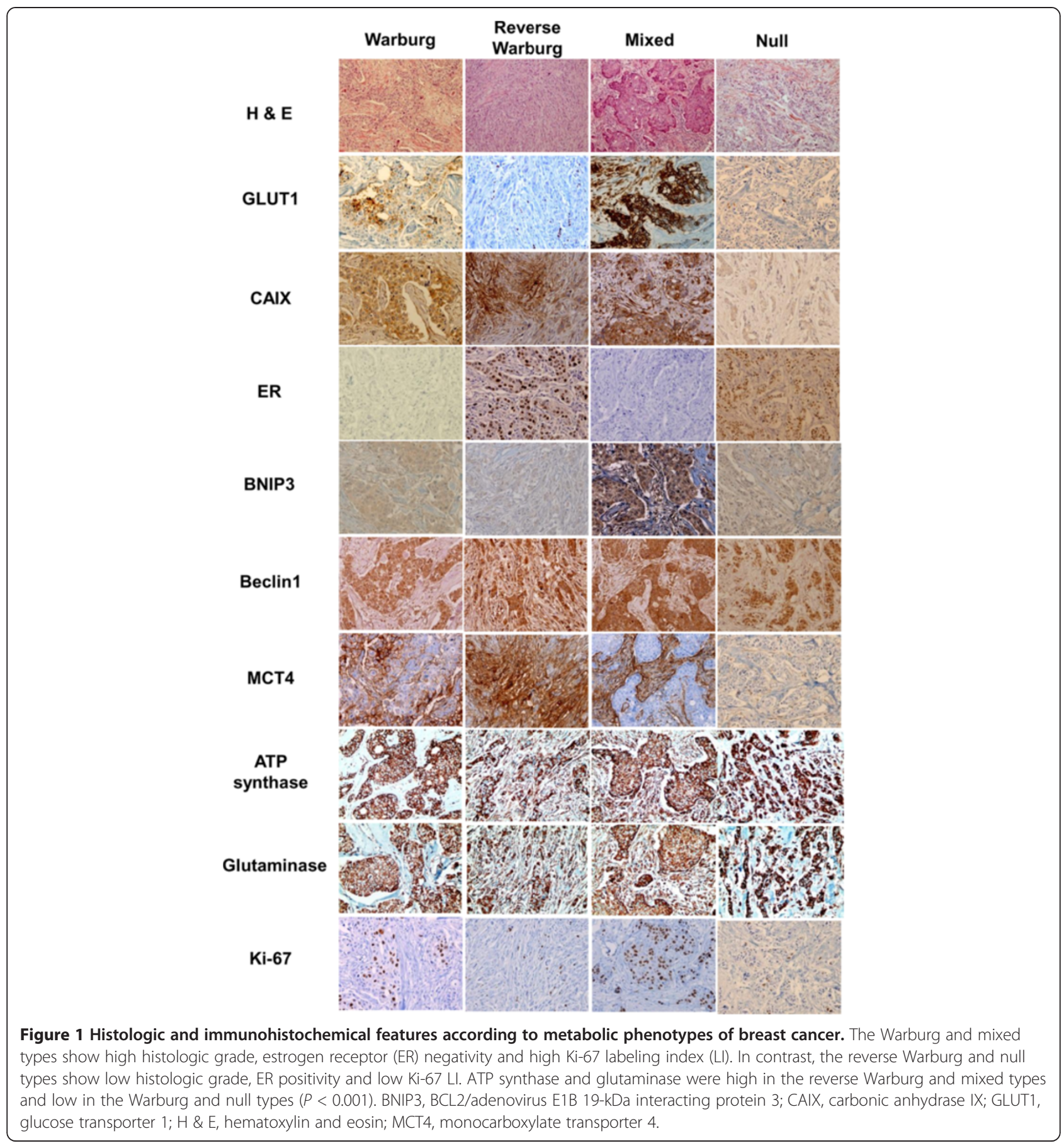

histologic grade, ER positivity, PR positivity and Ki-67 LI $(P<0.001)$. As both tumor cells and stromal cells are glycolytic in the mixed type and nonglycolytic in the null type, we speculate that the mixed type is a group of tumors with high metabolic activity and that the null type consists of tumors with lower metabolic activity. The results of the present study show that the mixed type had the highest percentage of activated autophagy, whereas the null type had the lowest percentage, thus supporting this hypothesis. In addition, different molecular subtypes of breast cancer were classified into different metabolic types. TNBC constituted the highest percentage of Warburg type and mixed type, whereas the luminal A type constituted the highest percentage of reverse Warburg type and null type $(P<0.001)$. Moreover, the Warburg type and the mixed type were classified into groups with a higher Ki-67 LI, whereas the reverse Warburg type and the null type were classified into groups with a lower Ki-67 LI $(P<0.001)$. 
Table 7 Univariate analysis of the expression of metabolism-related proteins in breast cancers and disease-free survival or overall survival by logrank test ${ }^{a}$

\begin{tabular}{|c|c|c|c|c|c|}
\hline \multirow{2}{*}{$\begin{array}{l}\text { Immunohistochemical } \\
\text { factors }\end{array}$} & \multirow{2}{*}{$\begin{array}{l}\text { Number of patients/ } \\
\text { recurrence/death }\end{array}$} & \multicolumn{2}{|c|}{ Disease-free survival } & \multicolumn{2}{|c|}{ Overall survival } \\
\hline & & $\begin{array}{l}\text { Mean survival } \\
(95 \% \mathrm{Cl}) \text {, months }\end{array}$ & $\overline{P \text {-value }}$ & $\begin{array}{l}\text { Mean survival } \\
(95 \% \mathrm{Cl}) \text {, months }\end{array}$ & $P$-value \\
\hline Glut-1 in tumor & & & 0.010 & & 0.023 \\
\hline Negative & $504 / 37 / 37$ & $128(125$ to 131$)$ & & 131 (128 to 134$)$ & \\
\hline Positive & $236 / 32 / 30$ & $119(112$ to 126$)$ & & $123(118$ to 128$)$ & \\
\hline Glut-1 in stroma & & & $\mathrm{n} / \mathrm{a}$ & & $\mathrm{n} / \mathrm{a}$ \\
\hline Negative & $724 / 69 / 67$ & $\mathrm{n} / \mathrm{a}$ & & $\mathrm{n} / \mathrm{a}$ & \\
\hline Positive & $16 / 0 / 0$ & $\mathrm{n} / \mathrm{a}$ & & $\mathrm{n} / \mathrm{a}$ & \\
\hline CAIX in tumor & & & 0.740 & & 0.222 \\
\hline Negative & $520 / 49 / 45$ & $126(122$ to 130$)$ & & 130 (127 to 132$)$ & \\
\hline Positive & $220 / 20 / 22$ & 108 (102 to 113$)$ & & $123(117$ to 130$)$ & \\
\hline CAIX in stroma & & & 0.927 & & 0.496 \\
\hline Negative & $627 / 59 / 56$ & $125(122$ to 129$)$ & & 129 (126 to 132$)$ & \\
\hline Positive & 113/10/11 & 103 (98 to 108) & & 116 (109 to 123$)$ & \\
\hline ATP synthase in tumor & & & 0.506 & & 0.936 \\
\hline Negative & $30 / 4 / 3$ & $102(90$ to 114$)$ & & 129 (117 to 141$)$ & \\
\hline Positive & $710 / 65 / 64$ & 125 (122 to 129$)$ & & $128(126$ to 131$)$ & \\
\hline ATP synthase in stroma & & & 0.783 & & 0.398 \\
\hline Negative & $570 / 53 / 50$ & 125 (121 to 129$)$ & & 129 (126 to 132$)$ & \\
\hline Positive & $170 / 16 / 17$ & $118(112$ to 124$)$ & & $122(115$ to 128$)$ & \\
\hline Glutaminase in tumor & & & 0.323 & & 0.164 \\
\hline Negative & $219 / 24 / 25$ & 123 (117 to 128$)$ & & 126 (120 to 131$)$ & \\
\hline Positive & $521 / 45 / 42$ & $126(122$ to 130$)$ & & 130 (127 to 133$)$ & \\
\hline Glutaminase in stroma & & & 0.554 & & 0.596 \\
\hline Negative & $495 / 49 / 48$ & 123 (119 to 128$)$ & & 128 (125 to 131$)$ & \\
\hline Positive & 245/20/19 & 127 (121 to 132$)$ & & 128 (124 to 133$)$ & \\
\hline BNIP3 in tumor & & & 0.004 & & 0.426 \\
\hline Negative & $504 / 59 / 50$ & 123 (119 to 127$)$ & & $128(124$ to 131$)$ & \\
\hline Positive & 236/10/17 & $123(119$ to 127$)$ & & 131 (126 to 135$)$ & \\
\hline BNIP3 in stroma & & & 0.191 & & 0.973 \\
\hline Negative & $700 / 68 / 64$ & 125 (121 to 128$)$ & & 128 (126 to 131$)$ & \\
\hline Positive & $40 / 1 / 3$ & $116(111$ to 121$)$ & & 121 (112 to 129$)$ & \\
\hline MCT4 in tumor & & & 0.550 & & 0.451 \\
\hline Negative & $540 / 49 / 47$ & $125(121$ to 129$)$ & & 129 (126 to 132$)$ & \\
\hline Positive & $200 / 20 / 20$ & $116(111$ to 121$)$ & & 126 (120 to 131$)$ & \\
\hline MCT4 in stroma & & & 0.673 & & 0.262 \\
\hline Negative & $418 / 42 / 44$ & 123 (118 to 127$)$ & & 127 (123 to 131) & \\
\hline Positive & $322 / 27 / 23$ & 128 (124 to 132$)$ & & 130 (126 to 133$)$ & \\
\hline Cytoplasmic beclin-1 & & & 0.169 & & 0.566 \\
\hline Negative & $406 / 46 / 38$ & 124 (119 to 128$)$ & & 129 (126 to 132$)$ & \\
\hline Positive & $334 / 23 / 29$ & 121 (118 to 124$)$ & & 126 (123 to 130$)$ & \\
\hline Nuclear beclin-1 & & & 0.157 & & 0.031 \\
\hline Negative & $666 / 66 / 66$ & 125 (121 to 128$)$ & & $128(125$ to 131$)$ & \\
\hline Positive & $74 / 3 / 1$ & 111 (106 to 115$)$ & & 136 (132 to 139$)$ & \\
\hline
\end{tabular}


Table 7 Univariate analysis of the expression of metabolism-related proteins in breast cancers and disease-free survival or overall survival by logrank test ${ }^{a}$ (Continued)

\begin{tabular}{|c|c|c|c|c|c|}
\hline $\mathrm{LC} 3 \mathrm{~A}$ in tumor & & & 0.085 & & 0.299 \\
\hline Negative & $669 / 59 / 59$ & $126(122$ to 129$)$ & & 129 (126 to 132$)$ & \\
\hline Positive & $71 / 10 / 8$ & 113 (103 to 122$)$ & & 124 (115 to 133$)$ & \\
\hline LC3A in stroma & & & 0.801 & & 0.541 \\
\hline Negative & $687 / 65 / 64$ & 125 (122 to 129$)$ & & $128(126$ to 131$)$ & \\
\hline Positive & $53 / 4 / 3$ & 65 (62 to 68$)$ & & 66 (64 to 68) & \\
\hline LC3B in tumor & & & 0.990 & & 0.271 \\
\hline Negative & $475 / 45 / 40$ & 125 (121 to 130$)$ & & 130 (127 to 133$)$ & \\
\hline Positive & $265 / 24 / 27$ & 118 (113 to 123$)$ & & 125 (120 to 130$)$ & \\
\hline LC3B in stroma & & & 0.481 & & 0.565 \\
\hline Negative & $688 / 66 / 64$ & $125(122$ to 129$)$ & & $128(126$ to 131$)$ & \\
\hline Positive & $52 / 3 / 3$ & 63 (60 to 66$)$ & & 64 (62 to 66) & \\
\hline Cytoplasmic p62 in tumor & & & 0.958 & & 0.528 \\
\hline Negative & $274 / 26 / 23$ & $121(112$ to 129$)$ & & $129(125$ to 133$)$ & \\
\hline Positive & $466 / 43 / 44$ & $126(122$ to 130$)$ & & 128 (125 to 131$)$ & \\
\hline Nuclear p62 in tumor & & & 0.210 & & 0.646 \\
\hline Negative & $532 / 55 / 51$ & 125 (122 to 129$)$ & & $128(125$ to 131$)$ & \\
\hline Positive & 208/14/16 & $117(110$ to 124$)$ & & $128(122$ to 133$)$ & \\
\hline Nuclear p62 in stroma & & & 0.720 & & 0.387 \\
\hline Negative & $512 / 48 / 45$ & $126(122$ to 130$)$ & & 129 (126 to 132$)$ & \\
\hline Positive & $228 / 21 / 22$ & 104 (99 to 109) & & 124 (118 to 130$)$ & \\
\hline Tumor phenotype & & & $<0.001$ & & $<0.001$ \\
\hline Luminal A & 298/15/14 & 130 (126 to 133$)$ & & 134 (131 to 137$)$ & \\
\hline Luminal B & 166/12/11 & $129(124$ to 134$)$ & & $130(124$ to 135$)$ & \\
\hline HER2 & $69 / 11 / 12$ & 111 (100 to 121$)$ & & 119 (108 to 130$)$ & \\
\hline TNBC & 207/31/30 & 116 (109 to 124$)$ & & $120(114$ to 126$)$ & \\
\hline Metabolic status & & & 0.037 & & 0.045 \\
\hline Warburg type & 298/38/36 & 119 (112 to 126$)$ & & 124 (119 to 128$)$ & \\
\hline Reverse Warburg type & $54 / 6 / 5$ & 90 (83 to 96$)$ & & 113 (106 to 121$)$ & \\
\hline Mixed type & $62 / 4 / 6$ & $105(100$ to 111$)$ & & 112 (99 to 126) & \\
\hline Null type & $326 / 21 / 20$ & 129 (126 to 133$)$ & & 132 (129 to 136$)$ & \\
\hline
\end{tabular}

This result is consistent with those of a former study on the reverse Warburg effect in which a luminal A breast cancer cell line, MCF-7, was used for in vitro study [6]. Thus, further in vitro studies should be carried out with various cell lines showing different molecular subtypes.

We identified the expression of a mitochondrial metabolism-related protein such as ATP synthase and glutaminase in the tumor and stroma in the present study. Notably, stromal expression of ATP synthase and glutaminase was high in the reverse Warburg type and mixed type and low in the Warburg type and null type $(P<0.001)$. We speculate that stroma showing glycolysis have high mitochondrial metabolic activity, as both the reverse Warburg and mixed types are subtypes of the glycolysis type of stroma by definition. Because it has also been reported that certain types of tumor generate ATP through glycolysis as well as through mitochondrial OXPHOS, the hypothesis that dual types of stromal metabolism via the glycolysis pathway and the mitochondrial pathway should be investigated further.

The present study shows that the Warburg type and mixed type consisted of metabolically active and biologically aggressive tumors, whereas the reverse Warburg type and null type consisted of metabolically inactive 

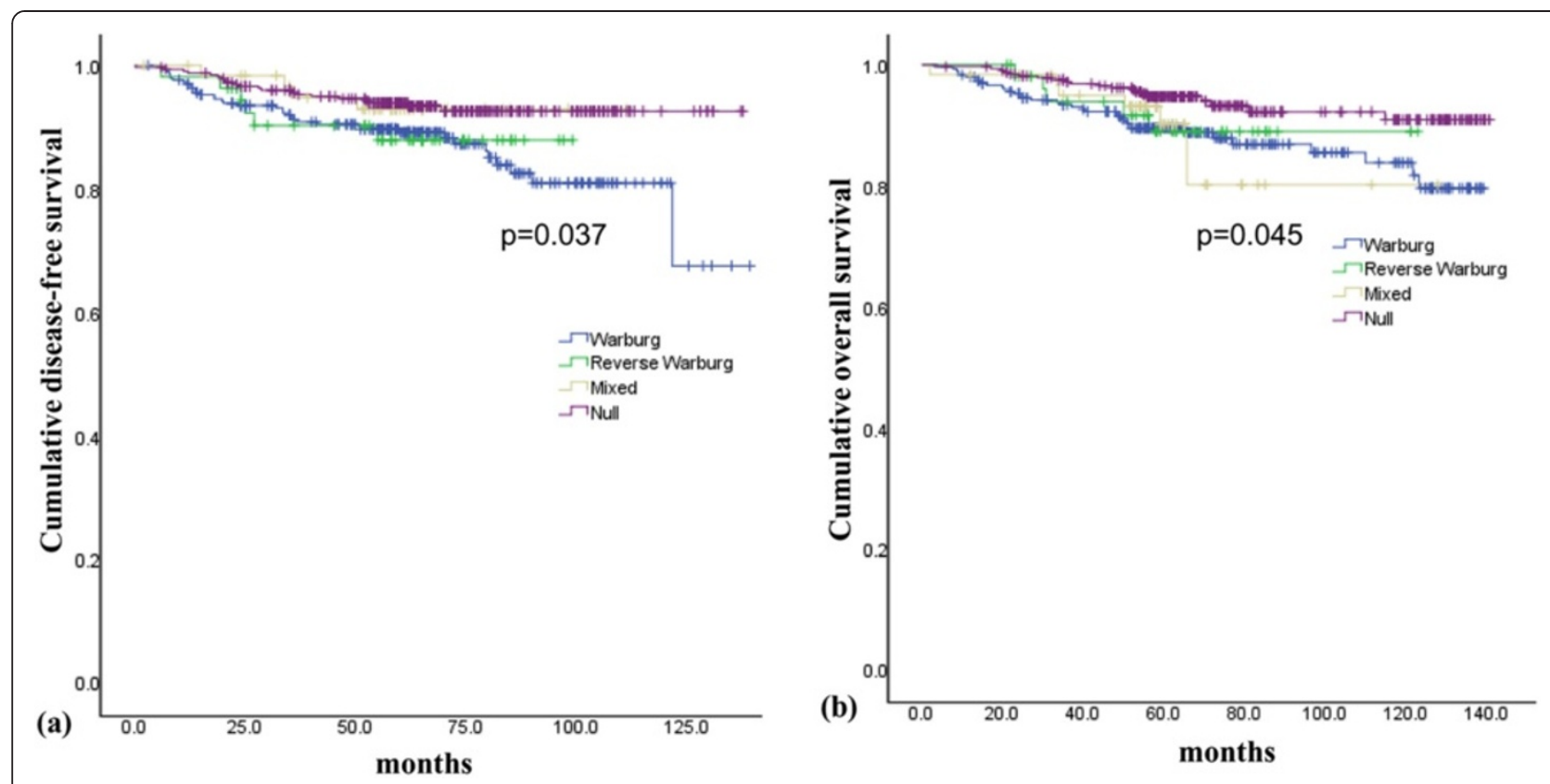

Figure 2 Disease-free survival (a) and overall survival curves (b) according to the metabolic phenotypes of breast cancer.

and biologically nonaggressive tumors. This finding suggests that glycolysis of tumors significantly affects their metabolic and biological characteristics. The association of Glut-1 with shorter DFS and OS in univariate analysis supports this hypothesis.

A potential limitation of this study is the use of TMA cores for analysis, which may not truly represent the whole tumor. Although it is a reasonable contention, given the well-known intrinsic heterogeneity of breast cancer, this limitation was overcome by using two 3-mm tissue cores because it was previously reported that TMA with two 0.6- $\mathrm{mm}$ cores were representative of standard full tissue sections in breast cancer [21].

Among the breast cancer subtypes, TNBC comprised $28 \%$ of the total cases in this study, which is higher than the previously reported $12 \%$ to $24 \%$. This difference can be attributed to possible differences in ethnic incidence, as reported previously, and to the overestimation of the true incidence potentially by the use of TMA containing part of the tumor, as we defined TNBC as all negative for ER, PR and HER2, which is similar to known phenomena of the discordance of ER, PR and HER2 expression between samples from core biopsy and excision [22-24]. Last, erroneous results of ER, PR and HER2 expression may affect the incidence, given that a $10 \%$ of false-negative rate and a $5 \%$ of false-positive rate were reported in ER expression, whereas a $4 \%$ of false-negative and false-positive cases were reported in HER2 [25]. Thus, cautious interpretation of the expression of those markers seems crucial, as misinterpretation of results may lead to the misclassification of the molecular subtypes.

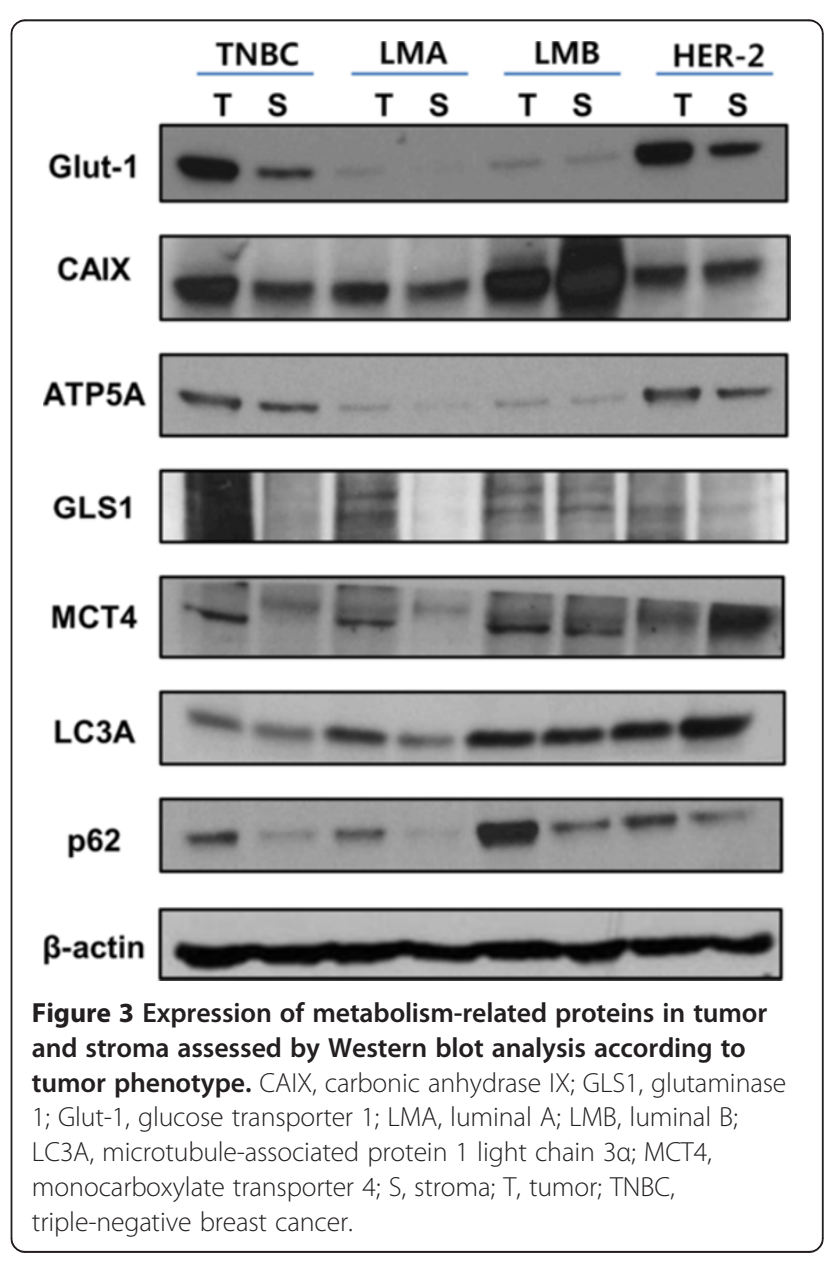




\section{Conclusion}

Breast cancer is heterogeneous in its metabolic status, and therefore it can be classified into various metabolic phenotypes. Specifically, the Warburg and mixed types had strong associations with TNBC, whereas the reverse Warburg type and the null type were associated with the luminal type, suggesting a correlation between metabolic phenotype and the biology of breast cancer.

\section{Abbreviations \\ ASCO: American Society of Clinical Oncology; CAF: Cancer-associated fibroblast; CAP: College of American Pathologists; FISH: Fluorescence in situ hybridization; H\&E: Hematoxylin and eosin; LI: Labeling indices; NO: Nitric oxide; OXPHOS: Oxidative phosphorylation; ROS: Reactive oxygen species; TMA: Tissue microarray; TNBC: Triple-negative breast cancer.}

\section{Competing interests}

The authors declare that they have no competing interests.

\section{Authors' contributions}

JC participated in the design of the study, performed the statistical analysis and drafted the manuscript. DHK carried out the immunoassays and Western blot analysis. WHJ participated in the study design. JSK conceived the study, participated in its design and coordination and helped to draft the manuscript. All authors read and approved the final manuscript.

\section{Acknowledgements}

This research was supported by the Basic Science Research Program through the National Research Foundation of Korea (NRF), funded by the Ministry of Education, Science and Technology (grant 2012R1A1A1002886).

\section{Author details}

'Department of Pathology, Yonsei University Wonju College of Medicine, Ilsan-dong 162, Wonju, Gangwon-do 220-701, South Korea. ${ }^{2}$ Department of Pathology, Yonsei University College of Medicine, 50 Yonsei-ro, Seodaemun-gu, Seoul 120-752, South Korea.

Received: 25 October 2012 Accepted: 18 July 2013

Published: 10 September 2013

\section{References}

1. Warburg O: On the origin of cancer cells. Science 1956, 123:309-314.

2. Bonuccelli G, Tsirigos A, Whitaker-Menezes D, Pavlides S, Pestell RG, Chiavarina B, Frank PG, Flomenberg N, Howell A, Martinez-Outschoorn UE, Sotgia F, Lisanti MP: Ketones and lactate "fuel" tumor growth and metastasis: evidence that epithelial cancer cells use oxidative mitochondrial metabolism. Cell Cycle 2010, 9:3506-3514.

3. Martinez-Outschoorn UE, Balliet RM, Rivadeneira DB, Chiavarina B, Pavlides S, Wang C, Whitaker-Menezes D, Daumer KM, Lin Z, Witkiewicz AK, Flomenberg N, Howell A, Pestell RG, Knudsen ES, Sotgia F, Lisanti MP: Oxidative stress in cancer associated fibroblasts drives tumor-stroma co-evolution: a new paradigm for understanding tumor metabolism, the field effect and genomic instability in cancer cells. Cell Cycle 2010, 9:3256-3276.

4. Pavlides $S$, Tsirigos A, Vera I, Flomenberg N, Frank PG, Casimiro MC, Wang C, Fortina P, Addya S, Pestell RG, Martinez-Outschoorn UE, Sotgia F, Lisanti MP: Loss of stromal caveolin-1 leads to oxidative stress, mimics hypoxia and drives inflammation in the tumor microenvironment, conferring the "reverse Warburg effect": a transcriptional informatics analysis with validation. Cell Cycle 2010, 9:2201-2219.

5. Pavlides S, Whitaker-Menezes D, Castello-Cros R, Flomenberg N, Witkiewicz AK, Frank PG, Casimiro MC, Wang C, Fortina P, Addya S, Pestell RG, Martinez-Outschoorn UE, Sotgia F, Lisanti MP: The reverse Warburg effect: aerobic glycolysis in cancer associated fibroblasts and the tumor stroma. Cell Cycle 2009, 8:3984-4001.

6. Martinez-Outschoorn UE, Pavlides S, Whitaker-Menezes D, Daumer KM, Milliman JN, Chiavarina B, Migneco G, Witkiewicz AK, Martinez-Cantarin MP, Flomenberg N, Howell A, Pestell RG, Lisanti MP, Sotgia F: Tumor cells induce the cancer associated fibroblast phenotype via caveolin-1 degradation: implications for breast cancer and DCIS therapy with autophagy inhibitors. Cell Cycle 2010, 9:2423-2433.

7. Martinez-Outschoorn UE, Trimmer C, Lin Z, Whitaker-Menezes D, Chiavarina B, Zhou J, Wang C, Pavlides S, Martinez-Cantarin MP, Capozza F, Witkiewicz AK, Flomenberg N, Howell A, Pestell RG, Caro J, Lisanti MP, Sotgia F: Autophagy in cancer associated fibroblasts promotes tumor cell survival: role of hypoxia, HIF1 induction and NFKB activation in the tumor stromal microenvironment. Cell Cycle 2010, 9:3515-3533.

8. Zu XL, Guppy M: Cancer metabolism: facts, fantasy, and fiction. Biochem Biophys Res Commun 2004, 313:459-465.

9. Moreno-Sánchez R, Rodríguez-Enríquez S, Marín-Hernández A, Saavedra E: Energy metabolism in tumor cells. FEBS J 2007, 274:1393-1418.

10. Kwon JE, Jung WH, Koo JS: Molecules involved in epithelial-mesenchymal transition and epithelial-stromal interaction in phyllodes tumors: implications for histologic grade and prognosis. Tumour Biol 2012, 33:787-798

11. Perou CM, Sørlie T, Eisen MB, van de Rijn M, Jeffrey SS, Rees CA, Pollack JR, Ross DT, Johnsen H, Akslen LA, Fluge O, Pergamenschikov A, Williams C, Zhu SX, Lønning PE, Børresen-Dale AL, Brown PO, Botstein D: Molecular portraits of human breast tumours. Nature 2000, 406:747-752.

12. Sørlie T, Perou CM, Tibshirani R, Aas T, Geisler $S$, Johnsen $H$, Hastie T, Eisen MB, van de Rijn M, Jeffrey SS, Thorsen T, Quist H, Matese JC, Brown PO, Botstein D, Lønning PE, Børresen-Dale AL: Gene expression patterns of breast carcinomas distinguish tumor subclasses with clinical implications. Proc Natl Acad Sci U S A 2001, 98:10869-10874.

13. Elston CW, Ellis IO: Pathological prognostic factors in breast cancer. I. The value of histological grade in breast cancer: experience from a large study with long-term follow-up. Histopathology 1991, 19:403-410.

14. Hammond ME, Hayes DF, Dowsett M, Allred DC, Hagerty KL, Badve S, Fitzgibbons PL, Francis G, Goldstein NS, Hayes M, Hicks DG, Lester S, Love R, Mangu PB, McShane L, Miller K, Osborne CK, Paik S, Perlmutter J, Rhodes A, Sasano H, Schwartz JN, Sweep FC, Taube S, Torlakovic EE, Valenstein P, Viale G, Visscher D, Wheeler T, Williams RB, Wittliff JL, Wolff AC: American Society of Clinical Oncology/College of American Pathologists guideline recommendations for immunohistochemical testing of estrogen and progesterone receptors in breast cancer. J Clin Oncol 2010, 28:2784-2795.

15. Wolff AC, Hammond ME, Schwartz JN, Hagerty KL, Allred DC, Cote RJ, Dowsett M, Fitzgibbons PL, Hanna WM, Langer A, McShane LM, Paik S, Pegram MD, Perez EA, Press MF, Rhodes A, Sturgeon C, Taube SE, Tubbs R, Vance GH, van de Vijver M, Wheeler TM, Hayes DF, American Society of Clinical Oncology; College of American Pathologists: American Society of Clinical Oncology/College of American Pathologists guideline recommendations for human epidermal growth factor receptor 2 testing in breast cancer. J Clin Oncol 2007, 25:118-145.

16. Goldhirsch A, Wood WC, Coates AS, Gelber RD, Thürlimann B, Senn HJ Panel members: Strategies for subtypes--dealing with the diversity of breast cancer: highlights of the St Gallen International Expert Consensus on the Primary Therapy of Early Breast Cancer 2011. Ann Oncol 2011, 22:1736-1747.

17. Bellot G, Garcia-Medina R, Gounon P, Chiche J, Roux D, Pouysségur J, Mazure NM: Hypoxia-induced autophagy is mediated through hypoxia-inducible factor induction of BNIP3 and BNIP3L via their BH3 domains. Mol Cell Biol 2009, 29:2570-2581.

18. Zhang H, Bosch-Marce M, Shimoda LA, Tan YS, Baek JH, Wesley JB, Gonzalez FJ, Semenza GL: Mitochondrial autophagy is an HIF-1-dependent adaptive metabolic response to hypoxia. J Biol Chem 2008, 283:10892-10903.

19. Pinheiro C, Sousa B, Albergaria A, Paredes J, Dufloth R, Vieira D, Schmitt F, Baltazar F: GLUT1 and CAIX expression profiles in breast cancer correlate with adverse prognostic factors and MCT1 overexpression. Histol Histopathol 2011, 26:1279-1286.

20. Reis-Filho JS, Tutt AN: Triple negative tumours: a critical review. Histopathology 2008, 52:108-118.

21. Camp RL, Charette LA, Rimm DL: Validation of tissue microarray technology in breast carcinoma. Lab Invest 2000, 80:1943-1949.

22. Park SY, Kim KS, Lee TG, Park SS, Kim SM, Han W, Noh DY, Kim SW: The accuracy of preoperative core biopsy in determining histologic grade, hormone receptors, and human epidermal growth factor receptor 2 status in invasive breast cancer. Am J Surg 2009, 197:266-269.

23. Chen X, Yuan Y, Gu Z, Shen K: Accuracy of estrogen receptor, progesterone receptor, and HER2 status between core needle and open excision biopsy in breast cancer: a meta-analysis. Breast Cancer Res Treat 2012, 134:957-967. 
24. Badve SS, Baehner FL, Gray RP, Childs BH, Maddala T, Liu ML, Rowley SC, Shak S, Perez EA, Shulman L, Martino S, Davidson NE, Sledge GW, Goldstein L, Sparano JA: Estrogen- and progesterone-receptor status in ECOG 2197: comparison of immunohistochemistry by local and central laboratories and quantitative reverse transcription polymerase chain reaction by central laboratory. J Clin Oncol 2008, 26:2473-2481.

25. Reddy JC, Reimann JD, Anderson SM, Klein PM: Concordance between central and local laboratory HER2 testing from a community-based clinical study. Clin Breast Cancer 2006, 7:153-157.

doi:10.1186/bcr3472

Cite this article as: Choi et al:: Metabolic interaction between cancer cells and stromal cells according to breast cancer molecular subtype. Breast Cancer Research 2013 15:R78.

\section{Submit your next manuscript to BioMed Central and take full advantage of:}

- Convenient online submission

- Thorough peer review

- No space constraints or color figure charges

- Immediate publication on acceptance

- Inclusion in PubMed, CAS, Scopus and Google Scholar

- Research which is freely available for redistribution 